\title{
Particle motion and diffusion at weak bed load: accounting for unsteadiness effects of entrainment and disentrainment
}

JENNY CAMPAGNOL, Former Assistant Researcher, Department of Civil and Environmental Engineering, Politecnico di Milano, Piazza L. da Vinci 32, 20133 Milan, Italy

Email: jenny.campagnol@polimi.it

ALESSIO RADICE (IAHR Member), Associate Professor, Department of Civil and Environmental Engineering, Politecnico di Milano, Piazza L. da Vinci 32, 20133 Milan, Italy

Email: alessio.radice@polimi.it (author for correspondence)

FRANCESCO BALLIO (IAHR Member), Professor, Department of Civil and Environmental Engineering, Politecnico di Milano, Piazza L. da Vinci 32, 20133 Milan, Italy

Email: francesco.ballio@polimi.it

VLADIMIR NIKORA (IAHR Member), Professor, School of Engineering, University of Aberdeen, Aberdeen AB24 $3 U E$, UK

Email:v.nikora@abdn.ac.uk

\begin{abstract}
A study of weak bed load over a plane bed is presented with a particular focus on the motion and diffusion of individual particles that were tracked along their movements. Grain position, velocity and acceleration were measured with particle-scale resolution; ensemble statistics over a significant sample of particle trajectories were then computed, enabling unsteady phases of particle motion (corresponding to entrainment and

disentrainment) to be identified. Based on the experimental findings, a physically-based conceptual model for particle kinematics consisting of an initial (entrainment) unsteady phase, a quasi-steady phase, and a final (disentrainment) unsteady phase is explored. The unsteadiness effects in particle dispersion due to entrainment and disentrainment, typically overlooked in previous studies, are explicitly quantified by analysing the time evolution of the particle position variance. Starting with a previously published model of particle diffusion, a refined model is proposed which explicitly accounts for the unsteadiness effects in particle motion.
\end{abstract}

Keywords: Bed load; disentrainment; entrainment; sediment acceleration; sediment diffusion; sediment motion; sediment velocity

\section{Introduction}

In spite of numerous sediment transport studies over several decades, the available models systematically fail in predict-ing bed-load transport rates with acceptable accuracy (e.g. Gomez \& Church, 1989; Heyman \& Ancey, 2014; Y. Martin, 2003; Recking, 2013). Shortfalls of existing models, together with recent progress in measurement techniques (particularly imagebased), have stimulated advanced studies aiming at deeper insights into physical mechanisms governing sediment motion at grain scale. New datasets and conceptual approaches, generated by these studies, highlight the effects of time and spatial fluctuations of sediment kinematic properties at the grain scale on the bulk sediment dynamics at larger scales (e.g. Ancey, Davison, Böhm, Jodeau, \& Frey, 2008; Furbish, Haff, Rose-berry, \& Schmeeckle, 2012). Thus, better understanding of the processes causing such fluctuations (i.e. particle-bed, particle-particle, and particle-fluid interactions) may lead to more accu-rate models of bulk sediment transport (Ballio \& Tait, 2012).

Earlier works dealing with the characterization of bed-load particle motion (sliding/rolling or saltating) typically quantified overall length, height and duration of grain trajectories or bulk velocity of moving particles (e.g. Drake, Shreve, Dietrich, Whiting, \& Leopold, 1988; Francis, 1973; Hu \& Hui, 1996;

This is an Accepted Manuscript version of the following article, accepted for publication in Journal of Hydraulic Research, 2015, 53:5, 633-648. It is deposited under the terms of the Creative Commons Attribution-NonCommercial License (http://creativecommons.org/licenses/by-nc/4.0/), which permits non-commercial re-use, distribution, and reproduction in any medium, provided the original work is properly cited http://dx.doi.org/10.1080/00221686.2015.1085920 
Julien \& Bounvilay, 2013; Lee \& Hsu, 1994; Niño \& Garcìa, 1998; Niño, Garcìa, \& Ayala, 1994; Ramesh, Kothyari, \& Murugesan, 2011). These integral quantities have been typically correlated with various bulk flow properties but the results from different studies are not consistent with each other (as shown, for example, by Campagnol, Radice, \& Ballio, 2012a). More information on grain kinematics is provided by the probability density function (PDF) of instantaneous particle velocities and its statistical moments (e.g. Fan, Zhong, Wu, Foufoula-Georgiou, \& Guala, 2014; Lajeunesse, Malverti, \& Charru, 2010; R.L. Martin, Jerolmack, \& Shumer, 2012; Roseberry, Schmeeckle, \& Furbish, 2012; Samson, Ippolito, Bideau, \& Batrouni, 1999). In general, the measured PDFs are characterized by a right-tail exponential decay and in most cases cannot be represented by simple equations (e.g. Gaussian, gamma, exponential) over the whole range of velocities.

Further information on bed particle dynamics can be obtained through the analysis of dispersive characteristics of grain motion using particle displacement data (e.g. Foufoula-Georgiou \& Stark, 2010; Nikora, Heald, Goring, \& McEwan, 2001). Measured or numerically simulated individual trajectories from a given homogeneous sediment transport field can be analysed by making their starting points coincide in space and time. This procedure allows the creation of ensembles of particle trajectories and, using them, assessment of the time evolution of the statistical moments of time-dependent coordinates (i.e. displacements) of moving particles. Let us define the longitudinal and transverse displacements from a starting point as $x_{p}\left(t_{p}\right)$ and $y_{p}\left(t_{p}\right)$, respectively, with $x_{p}\left(t_{p}=0\right)=y_{p}\left(t_{p}=0\right)=0$ result-ing from a superposition of the starting points of individual particle trajectories randomly sampled on the bed. The fluc-tuations around the ensemble averages $x_{p}\left(t_{p}\right)$ and $\overline{y_{p}}\left(t_{p}\right)$ are defined as $x_{p}$ ' $\left(t_{p}\right)$ and $y_{p}{ }^{\prime}\left(t_{p}\right)$. If particle motion is diffusive then the statistical moments of $x_{p}{ }^{\prime}\left(t_{p}\right)$ and $y_{p}{ }^{\prime}\left(t_{p}\right)$ can be expressed

as $\overline{x^{p} q} \propto t_{p}^{q \gamma_{x}(q)}$ and $\overline{y_{p}^{\prime q}} \propto t_{p}^{q \gamma_{y}(q)}$, where $q$ is the moment order and $\gamma_{x}(q)$ and $\gamma_{y}(q)$ are scaling exponents depending, in general, on the moment order $q$. For normal (Fickian) diffusion, we have $\gamma \equiv \gamma_{x}(q) \equiv \gamma_{y}(q) \equiv 0.5$ for even moments and $\gamma \equiv \gamma_{x}(q) \equiv \gamma_{y}(q) \equiv 0$ for odd moments (e.g. Bouchaud \& Georges, 1990). Anomalous diffusion arises when the Central Limit Theorem does not hold, which can happen due to either "broad" distributions (with diverging first or sec-ond moment) of particle motion characteristics and/or their "long-range" correlations (Bouchaud \& Georges, 1990; Hassan, Voepel, Schumer, Parker, \& Fraccarollo, 2013; Viswanathan, Raposo, Bartumeus, Catalan, \& da Luz, 2005; Wang, 1994; Weeks, Urbach, \& Swinney, 1996). The resulting anomalous diffusive process can be, respectively: ballistic $\left(\gamma_{x} \equiv \gamma_{y} \equiv 1\right)$, superdiffusive $\left(\gamma_{x}, \gamma_{y}>0.5\right)$ or sub-diffusive $\left(\gamma_{x}, \gamma_{y}<0.5\right)$ (Bouchaud \& Georges, 1990; Havlin \& Ben-Avraham, 2002; Metzler \& Klafter, 2004; Viswanathan et al., 2005).

Assuming diffusive properties of bed particle motion, Nikora et al. (2001) and Nikora, Habersack, Huber, and McEwan (2002) proposed a conceptual model involving at least three

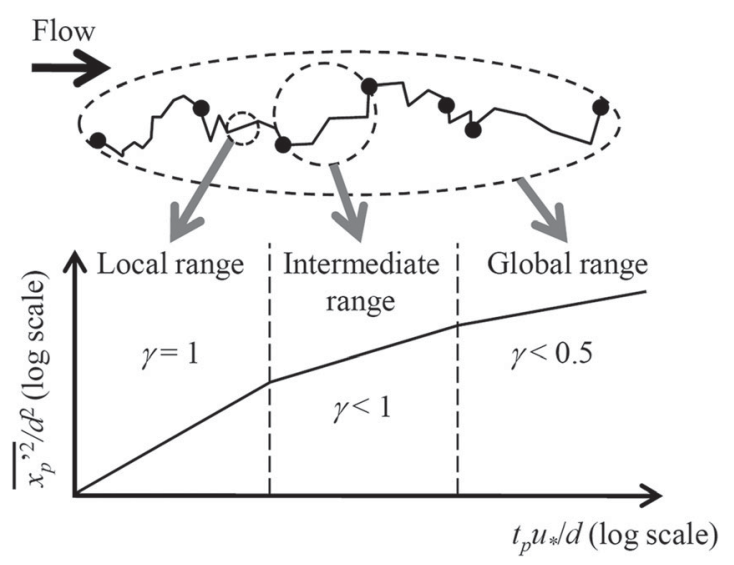

Figure 1 Conceptual model of Nikora et al. (2002) for a bed particle trajectory consisting of local, intermediate and global ranges of scales. Black circles define rest positions of a particle; $t_{p}$ is time from particle "release"; $u *$ is the shear velocity; $d$ is particle size; $x_{p}^{\prime 2}$ is the variance of streamwise displacement of particles; $\gamma$ is the diffusion exponent (it may vary and be different from the values / ranges shown in the sketch, depending on the bed topography, particle properties, and flow turbulence)

ranges of scales with distinctly different behaviour (Fig. 1): local, intermediate, and global. The local range corresponds to particle trajectory between two successive "significant collisions" with the bed (local trajectory). The intermediate range corresponds to particle trajectory between two particle rests (intermediate trajectory). The global range consists of many intermediate trajectories (global trajectory), similar to the intermediate trajectory that consists of many local trajectories. Within the local range, the diffusion process is assumed to be ballistic. Within the intermediate range, both turbulent flow fluc-tuations and bed-particle interactions affect particle motion that may result in either sub-diffusive, super-diffusive or normal dif-fusive behaviour depending on the dominating mechanism and bed topography structure. Finally, in the global range, particle behaviour is likely to be sub-diffusive as a result of a broad dis-tribution of resting times. Recent laboratory (Furbish, Ball, \& Schmeeckle, 2012; Furbish, Haff, et al., 2012; R.L. Martin et al., 2012; Roseberry et al., 2012; Zhang, Meerschaert, \& Packman, 2012) and field (Bradley, Tucker, \& Benson, 2010, using data of Sayre \& Hubbell, 1965; Zhang et al., 2012) experiments as well as detailed numerical simulations (Bialik, 2011; Bia-lik, Nikora, \& Rowiński, 2012) complement and expand Nikora et al.'s $(2001,2002)$ concept by providing results for a vari-ety of transport scenarios and scale ranges. A detailed table summarizing these findings is presented in Supplement A.

Most works on particle diffusion to date have considered the sediment transport process as an ensemble of homogeneous trajectories, overlooking potential effects of unsteady phases of particle motion. However, at the scale of intermediate trajectories the particle motion should be conceptually described in terms of three subsequent phases: entrainment unsteady phase, displacement quasi-steady phase, and final disentrain-ment unsteady phase (Drake et al., 1988). It has been observed 
that each of these stages may have different dynamics and duration depending on the motion mode and the leading physical factors (Furbish, Roseberry, \& Schmeeckle, 2012; Lajeunesse et al., 2010; Roseberry et al., 2012).

In this paper we present and analyse a set of laboratory measurements of grain kinematics under weak bed-load conditions, with the aim of characterizing the stochastic features of grain motion within the local and intermediate ranges. The novel components of the work include: (1) statistical characterization of particle motion at the smallest scales (local/grain); (2) analysis of the acceleration/deceleration patterns at the entrainment/disentrainment phases and of their effects on statistical (diffusive) characteristics of intermediate trajectories; and (3) refinement of Nikora et al.'s $(2001,2002)$ concept by accounting for the unsteadiness effects in particle motion.

\section{Experiments}

\subsection{Facility and sediment}

The experiments were performed at the Hydraulics Laboratory of the Politecnico di Milano in a $5.8 \mathrm{~m}$ long, $0.40 \mathrm{~m}$ wide, and $0.11 \mathrm{~m}$ high pressurized duct. Polybutylene terephthalate (PBT) plastic grains of the same size and quasi-prolate-ellipsoidal shape were used in the experiments as sediment particles. The measurements of the principal axes for 100 particles gave an average aspect ratio $A / C=2$ (where $A$ and $C$ are the lengths of the major and minor particle axes) and an equivalent diame-ter $d=\left(6 W_{s} / \pi\right)^{1 / 3}=3.0 \mathrm{~mm}$ (where $W_{s}$ is grain volume). The specific gravity of the particles was $\Delta=\left(\rho_{s}-\rho\right) / \rho=0.27$, where $\rho_{s}$ and $\rho$ are the grain and water densities, respectively. The use of low-density, artificial sediment has some advantages compared to natural sand. These advantages include reduced critical velocities, the possibility to paint particles more easily and, in addition, preserving the plastic walls of the duct that could be scratched by natural particles.

The $3.8 \mathrm{~m}$ long upstream section of the duct bed was made of steel plates covered by a layer of particles glued to their surface. The layer thickness was approximately two particle diameters. The remaining downstream part of the duct was equipped with a $2 \mathrm{~m}$ long recess section that could be either filled with loose particles (for mobile-bed runs, $\mathrm{MB}$ in the following) or covered with roughened plates, similar to the upstream part (for fixed-bed experiments, FB in the following). This part of the duct served as a working section for our experiments. The fixed-bed conditions approximated the roughness of a mobile bed reasonably well: the standard deviation of bed elevations, serv-ing as a characteristic roughness height (e.g. Nikora, Goring, \& Biggs, 1998), was equal to $2.21 \mathrm{~mm}$ for fixed bed and $2.55 \mathrm{~mm}$ for mobile bed. No bed-forms were present in the mobile-bed experiments.

Following Radice and Ballio (2008), the threshold flow rate $Q_{c}=1.0 \times 10^{-2} \mathrm{~m}^{3} \mathrm{~s}^{-1}$ for incipient particle motion was determined from preliminary tests as that inducing a dimensionless sediment discharge $q^{*}=q_{s} /\left(g \Delta d^{3}\right)^{0.5}=6 \times 10^{-5}$, where $q_{s}$ is the transport rate per unit width and $g$ is gravity acceleration. The sediment transport rate was measured by counting the sediment particles crossing a transverse line of the bottom surface. The target line was approximately in the middle of the working section; the particles were counted for several minutes (the time interval varied depending on the transport conditions). During the same tests, a relationship between the flow rate $Q$ and the transport rate $q_{s}$ was obtained for a range of $Q$ from $0.9 \times 10^{-2}$ $\mathrm{m}^{3} \mathrm{~s}^{-1}$ to $1.3 \times 10^{-2} \mathrm{~m}^{3} \mathrm{~s}^{-1}$.

In the following, $x, y, z$ axes are aligned with the longitudinal, transverse and vertical directions, respectively.

\subsection{Experimental scenarios, procedures, and preliminary tests}

The experiments were conducted for both FB and MB configurations. For each bed configuration, three different flow rates were used $\left(Q_{1}=1.11 \times 10^{-2} \mathrm{~m}^{3} \mathrm{~s}^{-1}, Q_{2}=1.22 \times 10^{-2} \mathrm{~m}^{3}\right.$ $\mathrm{s}^{-1}, Q_{3}=1.30 \times 10^{-2} \mathrm{~m}^{3} \mathrm{~s}^{-1}$, Table 1). Time-averaged vertical profiles of the streamwise velocity for each scenario were measured using two ultrasonic velocity profilers (UVP) deployed in the centre of the working section. The shear velocity and the hydrodynamic roughness length were then evaluated using the log-law equation:

$$
\frac{u(z)}{u_{*}}=\frac{1}{\kappa} \ln \frac{z}{z_{0}}
$$

where $u$ is the local time-averaged velocity, $u *$ is the shear velocity, $\kappa$ is the von Karman constant (a value of 0.40 was used), and $z_{0}$ is the hydrodynamic roughness length. The origin $z=0$ of the vertical coordinate was chosen based on the vertical profiles of echoes reflected from the bed and measured by the UVPs, i.e. at the top of the sediment layer. With this origin, Eq. (1) provided a reasonable fit to the data within the range $z=10-40$ $\mathrm{mm}$. The obtained estimates of the shear velocity and hydrodynamic roughness length, together with other relevant parameters, are given in Table 1 . The uncertainty in the $u *$ estimates, shown in Table 1, was quantified following McCuen (1993).

The experiments were designed to reproduce weak bed-load over a plane bed. Therefore, the maximum flow rate was limited to prevent generation of bed-forms. As a result, the maximum flow rate was set up to be only $17 \%$ higher than its minimum value. However, the quantity $\left(Q-Q_{c}\right) / Q_{c} \approx\left(u *-u *_{c}\right) / u *_{c}$ ( $u *_{c}$ is the critical shear velocity) differed by $270 \%$, with a five-fold variation in the dimensionless sediment discharge $q^{*}$ (Table 1). Table 1 shows that such a significant difference was essentially due to the corresponding difference in the concentration $C_{m}$ of moving particles, defined as the ratio of the time-average volume of moving particles to the volume of a single-particle sediment layer. The sediment transport mode was mostly rolling, with limited saltation.

To ensure the stability of the mean bed elevation in MB runs, a layer of loose grains was spread over the entire bed prior to the 
Table 1 Hydrodynamic conditions of the experimental tests

\begin{tabular}{|c|c|c|c|}
\hline Flow rate, $Q\left(\mathrm{~m}^{3} \mathrm{~s}^{-1}\right)$ & $1.11 \times 10^{-2}$ & $1.22 \times 10^{-2}$ & $1.30 \times 10^{-2}$ \\
\hline Bulk flow velocity, $U\left(\mathrm{~m} \mathrm{~s}^{-1}\right)$ & 0.25 & 0.28 & 0.30 \\
\hline $\begin{array}{l}\text { Shear velocity and hydrodynamic roughness length }-\mathrm{FB} \\
u *\left(\mathrm{~m} \mathrm{~s}^{-1}\right) \\
z_{0}(\mathrm{~mm})\end{array}$ & $\begin{array}{l}0.016 \pm 0.002 \\
2.0 \times 10^{-2}\end{array}$ & $\begin{array}{l}0.018 \pm 0.002 \\
3.0 \times 10^{-2}\end{array}$ & $\begin{array}{l}0.023 \pm 0.003 \\
8.1 \times 10^{-2}\end{array}$ \\
\hline $\begin{array}{l}\text { Shear velocity and hydrodynamic roughness length }-\mathrm{MB} \\
u *\left(\mathrm{~m} \mathrm{~s}^{-1}\right) \\
z_{0}(\mathrm{~mm})\end{array}$ & $\begin{array}{l}0.017 \pm 0.003 \\
2.9 \times 10^{-2}\end{array}$ & $\begin{array}{l}0.020 \pm 0.005 \\
4.0 \times 10^{-2}\end{array}$ & $\begin{array}{l}0.020 \pm 0.003 \\
2.6 \times 10^{-2}\end{array}$ \\
\hline$Q / Q_{c}$ & 1.11 & 1.22 & 1.30 \\
\hline Dimensionless sediment transport rate per unit width, $q^{*}$ & $1.0 \times 10^{-3}$ & $3.0 \times 10^{-3}$ & $4.7 \times 10^{-3}$ \\
\hline $\begin{array}{l}\text { Concentration of moving grains }-\mathrm{FB} \\
C_{m}(-)\end{array}$ & $2.1 \times 10^{-4}$ & $5.3 \times 10^{-4}$ & $7.2 \times 10^{-4}$ \\
\hline $\begin{array}{l}\text { Concentration of moving grains }-\mathrm{MB} \\
C_{m}(-)\end{array}$ & $1.2 \times 10^{-4}$ & $5.3 \times 10^{-4}$ & $7.2 \times 10^{-4}$ \\
\hline Flow Reynolds number, $\mathrm{R}=U H / v$ & $1.4 \times 10^{4}$ & $1.5 \times 10^{4}$ & $1.6 \times 10^{4}$ \\
\hline $\begin{array}{l}\text { Particle Reynolds number, } \mathrm{R}^{*}=u^{*} d / v \\
\text { (values of } u * \text { refer to } \mathrm{FB} \text { conditions) }\end{array}$ & $48 \pm 6$ & $54 \pm 6$ & $69 \pm 9$ \\
\hline
\end{tabular}

Note: $v$ is fluid viscosity and $H$ is half of the duct height

experiments and, during the runs, an automatic feeder was used to supply the sediment with a rate equal to the transport capacity determined during the preliminary tests. The instantaneous bed elevations were measured at two positions located at the centreline within the working area (at $4.2 \mathrm{~m}$ and $5.2 \mathrm{~m}$ from the inlet) using an echo-sounder with a vertical resolution of $2 \mathrm{~mm}$. The standard deviations of bed elevation time series measured during the tests were in all cases lower than $2 \mathrm{~mm}$, indicating the stationarity of the mobile-bed conditions. The particle Reynolds number was within 50-60, suggesting a transitional roughness regime (Table 1).

The particle motion was filmed from above with a CCD camera at a frequency of $32 \mathrm{~Hz}$. The focus rectangular window of the camera was elongated in the streamwise direction, with its centre at $4.5 \mathrm{~m}$ downstream of the inlet and its area of approximately $0.5 \times 0.36 \mathrm{~m}^{2}$ (resolution of 1.9 pixel mm $\mathrm{mm}^{-1}$ ). The sequences of images were collected with the aim of identifying intermediate trajectories though image processing (IP). For the investigated transport conditions, all the grains stopped at least twice within the focus area, thus allowing sampling and analysis of complete intermediate particle trajectories. To reduce the errors in IP, a mixture of black and white particles was fed to the duct and only the white grains, that were $5 \%$ of the total supply rate, were tracked. The main steps of the IP (fully described in Campagnol et al., 2013) included particle identification and tracking, extraction and validation of intermediate trajectories. White particles were identified by suppressing background, converting frames from greyscale to binary representation, and then filtering image noise. Particle tracking was based on a minimum-distance approach that was appropriate given the low percentage of white particles. In the next step, each particle was first given motion or no-motion label at each time instant, based on the following criterion: a particle at a

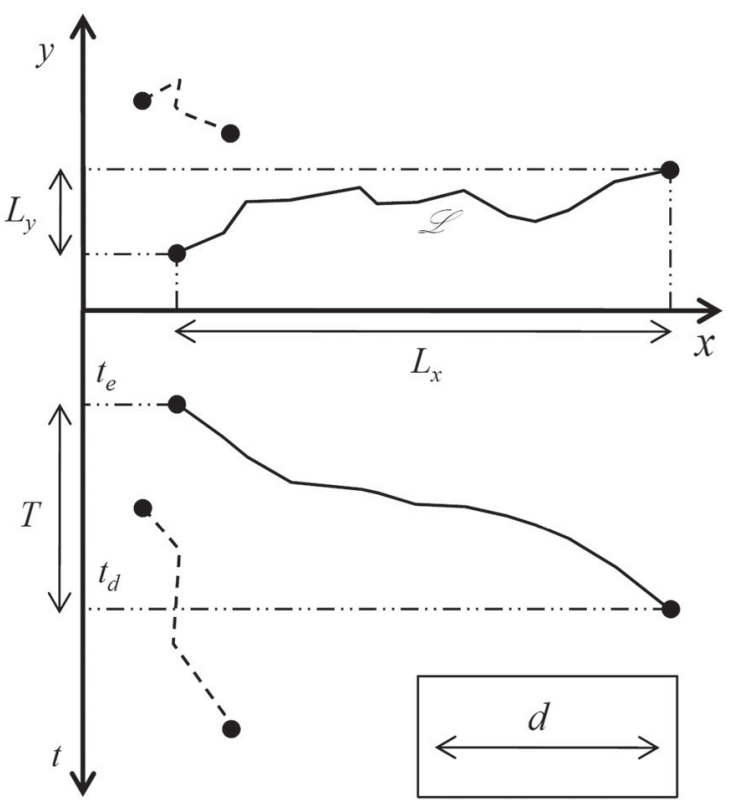

Figure 2 Spatial and temporal history of a moving particle $\left(L_{x}>d\right.$, solid) and a vibrating particle $\left(L_{x}<d\right.$, dashed). For the entrained particle definitions of $\mathscr{L}, L_{x}, L_{y}, t_{e}, t_{d}$ and $T$ are also given

certain $x$ position was considered to be at motion if it did not keep the same $x$ (or lower) in the following frames. Finally, intermediate trajectories were extracted and underwent visual inspection to ensure the best possible data quality. If tracking errors were detected, the inspected trajectory was not validated and was thus excluded from the sample. In addition, intermediate particle trajectories were included in the ensemble only if a particle travelled a distance of at least $1 d$ along the streamwise direction. Otherwise, a particle was classified as a vibrating one and its trajectory was not considered for the present analysis (Fig. 2). The time-dependent coordinates of moving grains 
Table 2 Statistical characteristics of intermediate trajectory duration $T$

\begin{tabular}{|c|c|c|c|c|c|c|}
\hline & \multicolumn{2}{|c|}{$Q_{1}=1.11 \times 10^{-2}\left(\mathrm{~m}^{3} \mathrm{~s}^{-1}\right)$} & \multicolumn{2}{|c|}{$\underline{Q_{2}}=1.22 \times 10^{-2}\left(\mathrm{~m}^{3} \mathrm{~s}^{-1}\right)$} & \multicolumn{2}{|c|}{$Q_{3}=1.30 \times 10^{-2}\left(\mathrm{~m}^{3} \mathrm{~s}^{-1}\right)$} \\
\hline & FB & $\mathrm{MB}$ & FB & MB & FB & MB \\
\hline Number of trajectories, $N_{t r}$ & 743 & 552 & 993 & 811 & 1229 & 1106 \\
\hline \multicolumn{7}{|c|}{ Statistics for intermediate trajectory duration, $T$ (s) } \\
\hline $\bar{T}$ & 0.57 & 0.61 & 0.55 & 0.55 & 0.55 & 0.58 \\
\hline$\sigma_{T}(\mathrm{~s})$ & 0.40 & 0.42 & 0.35 & 0.38 & 0.38 & 0.43 \\
\hline$S k_{T}$ & 1.71 & 1.61 & 1.78 & 1.97 & 1.87 & 2.03 \\
\hline$K u_{T}$ & 4.01 & 3.17 & 4.94 & 6.70 & 5.21 & 6.35 \\
\hline
\end{tabular}

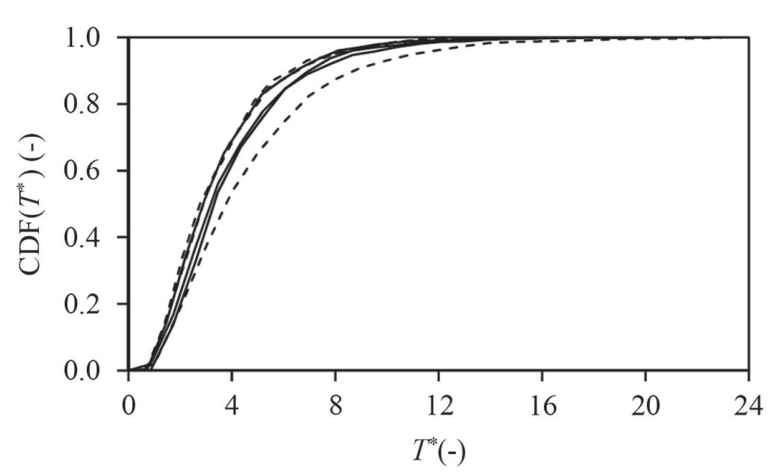

Figure 3 Cumulative distribution function (CDF) of the dimensionless duration of intermediate particle trajectories, $T^{*}=T u * / d$, for the tested scenarios (FB and MB tests are in dashed and solid lines, respectively)

within their intermediate trajectories were the final output of the IP. For each experimental condition, more than 500 intermediate trajectories were identified and quantified (Table 2). The first- to fourth-order moments of the duration of inter-mediate particle trajectories $T$ (defined in Fig. 2) are given in Table 2, while Fig. 3 depicts the cumulative distribution func-tion (CDF) of $T^{*}=T u_{*} / d$ for all experimental scenarios. The data in Table 2 and Fig. 3 show that $T$ was not strongly depen-dent on the bed configuration and flow conditions. The average duration of particle trajectories ranged from $0.55 \mathrm{~s}$ to $0.61 \mathrm{~s}$.

\section{Particle motion within intermediate trajectories}

\subsection{Measured variables and reference times}

The length of an intermediate trajectory is defined by $\mathscr{L}$ while the trajectory projections on the streamwise and transverse axes are defined as $L_{x}$ and $L_{y}$, respectively (Fig. 2). The position (i.e. coordinates $x$ and $y$ ) of a moving particle was tracked with a temporal resolution imposed by the camera acquisition frequency (32 fps). Consistently with the definitions above, we also delineate the particle displacement $\Delta \mathscr{L}$ and its projections $\Delta L_{x}$ and $\Delta L_{y}$ that correspond to the time interval separating two successive movie frames. The projections $\Delta L_{x}$ and $\Delta L_{y}$ were used to compute instantaneous particle velocity components $v_{x}$ and $v_{y}$. Then, the streamwise component $a_{x}$ of the instantaneous particle acceleration was computed using the $v_{x}$ data. In the following, the ensemble statistics of the grain kinematic characteristics will be presented as a function of travel time. The word "ensemble" refers to the sample of measured trajectories. The accuracy of instantaneous particle velocity is determined by the combination of the image resolution and frame rate, with the measurement error being inversely proportional to the time interval between frames. Reasonably assuming the uncertainty in the determination of the particle position to be one pixel, the instantaneous particle velocity would be estimated with uncertainty of $17 \mathrm{~mm} \mathrm{~s}^{-1}$, reducing to $0.72 \mathrm{~mm} \mathrm{~s}^{-1}$ when considering ensemble-averaged velocities over a sample of 552 values (which was the minimum sample size used in this work for ensemble averaging; Table 2). As an increase in the camera frame rate would inevitably increase the uncertainty level, we resolved to use $32 \mathrm{fps}$ as a compromise between conflicting requirements for minimal uncertainty level and for high temporal resolution.

Within the intermediate range of scales the particle motion is delimited by instants of entrainment, $t_{e}$, and disentrainment, $t_{d}$ (Fig. 2, with $t$ as time). These instants differ from one trajectory to another since they correspond to motion events spread in time and space. For the statistical analysis, we used two reference times. On one hand, we combined the trajectories together by superimposing their entrainment instants and defining a new time coordinate $t_{p e}=\left(t-t_{e}\right)$ corresponding to time after entrainment. This allowed us to explore the entrainment dynamics and its role in particle dispersion. On the other hand, we also studied the ensembles of trajectories obtained by superimposing their disentrainment instants and defining $t_{p d}=\left(t-t_{d}\right)$ as the time from disentrainment (note that values of $t_{p d}$ are intrinsically negative). Figure 4 illustrates the applied procedure, also showing relative coordinates $x_{p e}$ and $x_{p d}$ that are defined similarly to $t_{e}$ and $t_{d}\left(y_{p e}\right.$ and $y_{p d}$ for the $y$ coordinate are defined in the same way, not shown).

\subsection{Particle kinematics: entrainment (acceleration) phase and quasi-steady phase}

\section{Probability distribution of particle velocity}

Figure 5a shows the typical PDFs of the streamwise particle velocity $v_{x}$ at given time instants after entrainment for test MB$\mathrm{Q}_{3}$. As one can note, the likelihood of low values of particle 

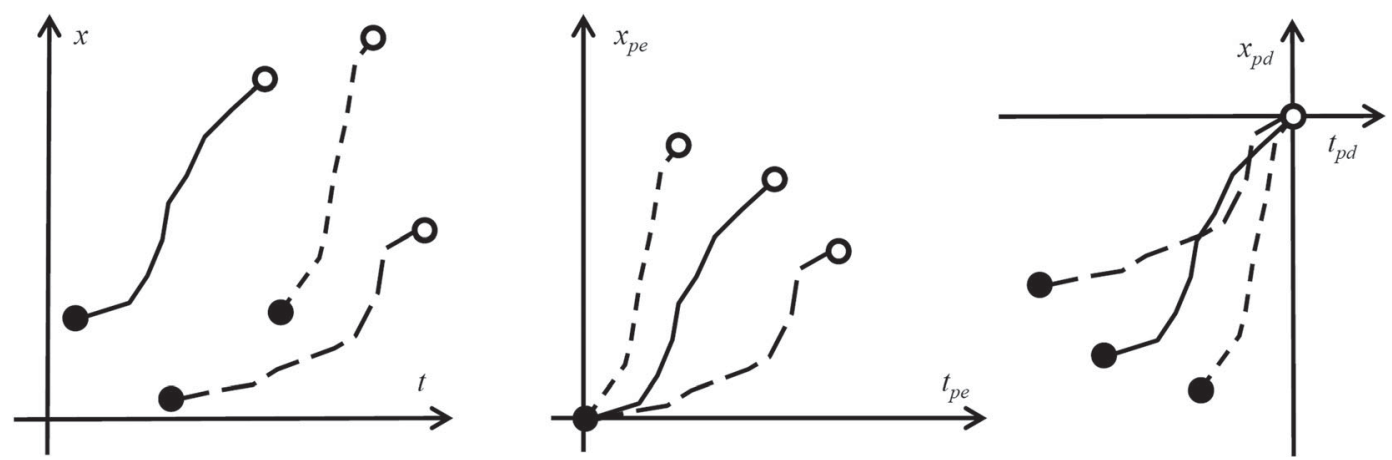

Figure 4 Sketches of intermediate trajectories with entrainments (filled circles) and disentrainments (hollow circles) are shown on the left. Trajectories superimposed using entrainments and disentrainments as reference points are shown in the middle and on the right, respectively

velocity decreases with increase in $t_{p e}$ : a clear transition in the PDF shape is evident at $t_{p e}=0.1 \mathrm{~s}$, above which the mode of the PDF is no longer at the lowest measured velocity value. For times longer than $t_{p e} \cong(0.1-0.3) \mathrm{s}$, the PDFs are similar to each other, suggesting that at sufficiently long travel times the particle motion tends to be (quasi-)steady. The exceedance distribution functions (EDF $=1-\mathrm{CDF}$ ) in Fig. 5b show that their decay at sufficiently large $t_{p e}$ is approximately exponential, as earlier highlighted by Furbish and Schmeeckle (2013). Figure $5 \mathrm{c}$, depicting the PDFs of $v_{x} / u *$ for all experimental scenarios at two dimensionless instants $t_{p e} u_{*} / d=0.5$ and $t_{p e} u_{*} / d=3$, reveals a reasonable collapse of PDFs at the same $t_{p e} u_{*} / d$, reflecting their independence of transport capacities (Table 1). Self-similarity of velocity distributions in Fig. 5c in respect to the transport intensity is consistent with previous studies (e.g. Ancey et al., 2008; Campagnol, Radice, \& Ballio, 2012b; Drake et al., 1988; Niño \& Garcìa, 1998; Niño et al., 1994; Radice \& Ballio, 2008; Radice, Ballio, \& Nikora, 2010). In addition, this result, as well as other findings below, strongly suggests that observations for FB conditions may well represent also the MB scenarios. The PDF for the transverse particle velocity $v_{y}$ exhibits a bell shape (Fig. 5d) as was also reported by Lajeunesse et al. (2010). Overall, the time evolution of the probability density function of $v_{y}$ demonstrates properties similar to those found for $v_{x}$. Particularly, there is a transition at $t_{p e}$ $\cong(0.1-0.3) \mathrm{s}$ from an unsteady phase of particle motion at small travel times to the (quasi-)steady phase at larger $t_{p e}$. Similar to that of $v_{x}$, the EDF of $v_{y}$ also features an exponential decay at sufficiently large $t_{p e}$ (not shown here).

\section{Statistical moments of particle velocity}

The ensemble-averaged velocity at time $t_{p e}$ after entrainment was computed as:

$$
\overline{v_{i}}\left(t_{p e}\right)=\frac{1}{N} \sum_{\mathrm{n}=1}^{N} v_{i \mathrm{n}}\left(t_{p e}\right)
$$

where $v_{i}$ denotes either $v_{x}$ or $v_{y}$, overbar denotes the ensemble averaging, and $N$ is the number of trajectories available for averaging at $t_{p e}$. Note that $N \leq N_{t r}$ as only trajectories with duration $T \geq t_{p e}$ can be used for averaging at $t_{p e}$, where $N_{t r}$ is the total number of trajectories collected for each test (Table 2). Ensemble-averaged particle acceleration and travel distances over $t_{p e}$ were computed similarly. Figure 6a presents, as an example, the ensemble averaging of the streamwise instantaneous particle velocity for test $\mathrm{MB}-Q_{3}$. As with PDFs, the time evolution of the ensemble-averaged velocity $\overline{v_{x}}$ clearly reveals two distinctly different phases of particle motion: (1) an unsteady (acceleration) phase at $t_{p e}<0.35 \mathrm{~s}$; and (2) a (quasi-)steady phase at $t_{p e}>0.35 \mathrm{~s}$ (Fig. 6a). The streamwise particle velocity in the (quasi-)steady phase (Figs 5a and 6a) appears to be much lower than the water velocity ( $\cong 200 \mathrm{~mm}$ $\mathrm{s}^{-1}$ ) just above the layer of moving particles. This difference is consistent with a prevalence of rolling / sliding over saltation that was indeed observed visually.

Figure $6 \mathrm{~b}$ depicts the time evolution of the key ensembleaveraged kinematic parameters of entrained particles for test MB- $Q_{3}$. The prevalence of streamwise particle motion over its transverse motion can be recognized considering that the streamwise travel distance $\overline{L_{x p e}}$ at time $t_{p e}$ is not much different from the along-trajectory distance $\overline{\mathscr{L}_{p e}}$ (Fig. 6b). The particle acceleration attains a maximum at the start of motion but then sharply decreases before increasing again, with a second peak occurring at around $t_{p e} \cong 0.1 \mathrm{~s}$. This behaviour may be interpreted assuming an entrainment mechanism by rollover with a typical duration $\tau_{r} \cong 0.1 \mathrm{~s}$ (note that $\tau_{r}$ is obtained using ensemble-averaged data, not from individual trajectories; throughout the entire manuscript, quantities indicated with $\tau$ and a subscript will indicate time lapses obtained from ensemble-averaged trajectories). The rollover mechanism, visually observed in our laboratory experiments, appeared to be similar to that described earlier by Drake et al. (1988): "generally involved movement through the gap between two of the surrounding bed particles or, less commonly, directly over the top of one". When the exposure of the entrained grain to the flow becomes higher, the sharply increased fluid drag leads to increase in acceleration forming the second maximum (denoted in Fig. $6 \mathrm{~b}$ with $\tau_{r}$ ). During the rollover, a particle typically travels a distance of $\overline{\mathscr{L}_{p e}} \cong 1 d$ (Fig. 6b). After the entrainment, the particle acceleration progressively diminishes until a 

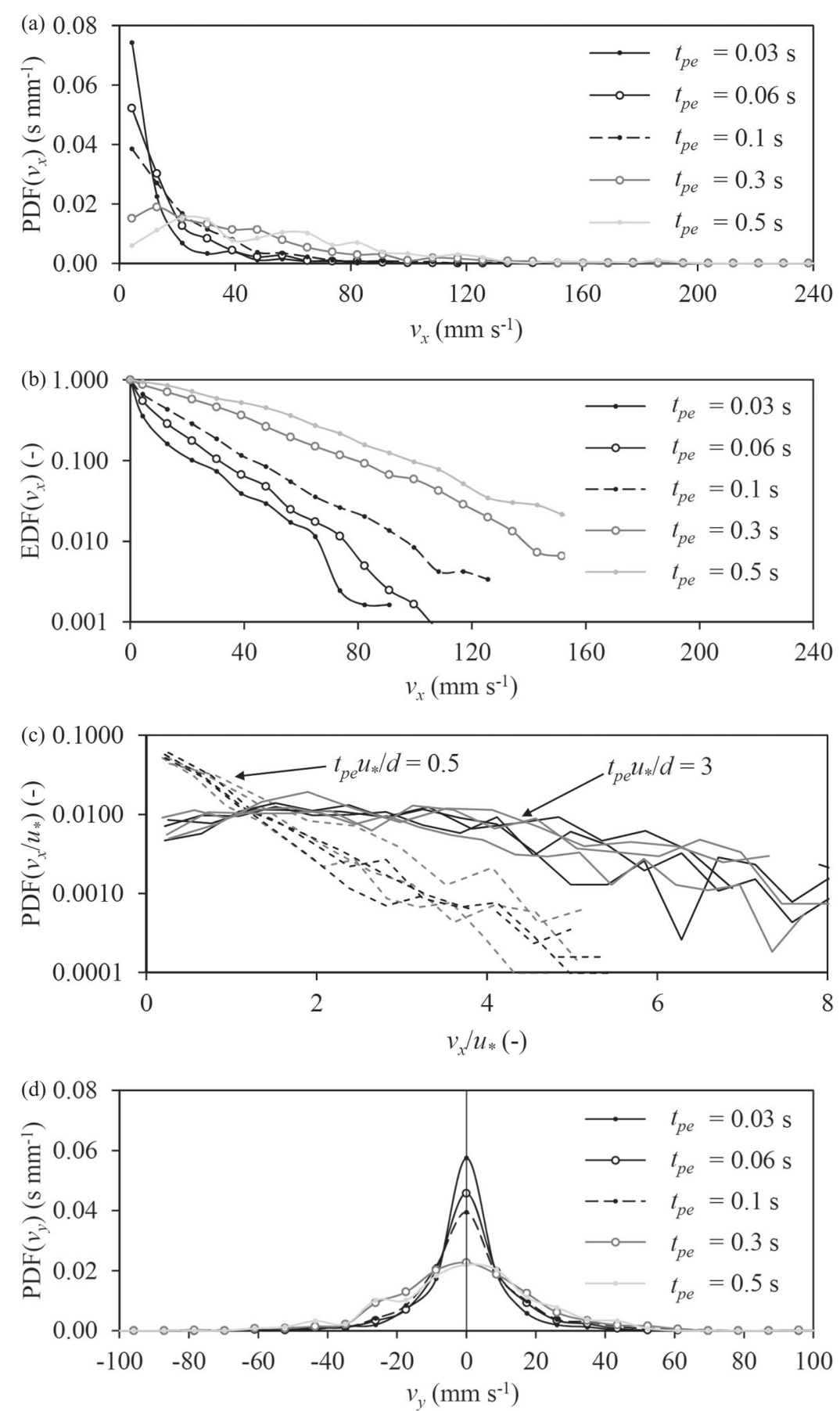

Figure 5 Distributions of instantaneous velocity components at given time instants after entrainment: (a) probability density function (PDF) and (b) exceedance distribution function (EDF) of $v_{x}$ for test MB- $Q_{3}$; (c) PDF of $v_{x}$ at $t_{p e} u * / d=0.5$ (dashed lines) and $t_{p e} u * / d=3$ (continuous lines) for all tests, FB in grey and MB in black; (d) PDF of $v_{y}$ for test MB- $Q_{3}$. Note log scales on vertical axes for plots (b) and (c)

quasi-steady state of motion is reached. As already mentioned, the total duration $\tau_{a}$ of the unsteady (acceleration) phase is $\tau_{a}$ $\cong 0.35 \mathrm{~s}$ which corresponds to a travel distance around three particle diameters (Fig. 6b).

The results reported above for $\mathrm{MB}-Q_{3}$ are representative of all other experiments. Figure $6 \mathrm{c}$ shows the dimensionless time evolution of $\overline{v_{x}}$ and $\overline{a_{x}}$ for all studied scenarios. In agreement with the trend suggested by the PDFs of particle velocity, in all tests an initial unsteady (acceleration) phase is followed by a quasi-steady phase. Moreover, the acceleration data suggest that the unsteady phase actually includes two sub-phases: a "rollover" sub-phase (from the beginning of motion to the second acceleration peak) and a "reducing acceleration" sub-phase (from the second acceleration peak to the quasi-steady state) (Fig. 6). The combination of these sub-phases can be referred to as the initial unsteady phase or, simply, acceleration phase. The transition from the unsteady (acceleration) phase to the quasisteady phase is likely asymptotic and thus identification 

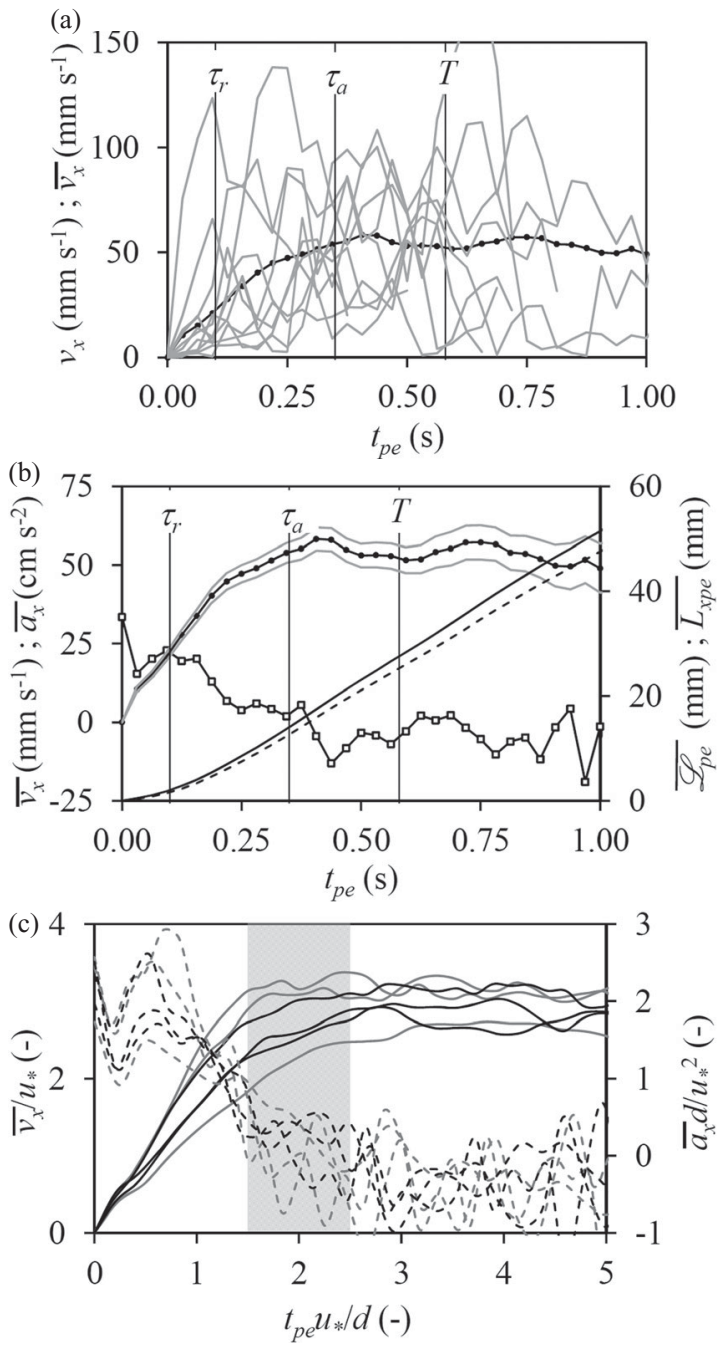

Figure 6 Time evolution of the kinematic characteristics of moving particles: (a) velocity $v_{x}$ for some sample trajectories (grey lines) and its ensemble-averaged value (black line with circles) for test MB- $\mathrm{Q}_{3}$; (b) ensemble-averaged $\overline{v_{x}}$ (black line with circles) with $99 \%$ confidence intervals (grey lines), $\overline{a_{x}}$ (line with hollow squares), and ensemble-averaged travel distances within $t_{p e}: \overline{\mathscr{L}_{p e}}$ (along the trajectory, continuous black line), and $\overline{L_{x p e}}$ (projection on the $x$-axis, dashed black line) for test $\mathrm{MB}-\mathrm{Q}_{3}$; (c) dimensionless time evolution of $\overline{v_{x}} / u_{*}$ (continuous) and $\overline{a_{x}} d / u_{*}^{2}$ (dashed) for all tests (FB in grey and MB in black). Vertical lines in (a) and (b) denote the "rollover" sub-phase (from 0 to $\tau_{r}$ ), the "decreasing acceleration" sub-phase (from $\tau_{r}$ to $\tau_{a}$ ), and the mean trajectory duration $(T)$; the grey strip in (c) defines a range for dimensionless duration of the unsteady (acceleration) phase $\tau_{a} *$ of particle motion

of the boundary between them is not straightforward. Nevertheless, Fig. $6 \mathrm{c}$ indicates that the transition occurs within a range of $\tau_{a} *=\tau_{a} u * / d \cong 1.5-2.5$ (shown in grey).

The time evolution of the second- to fourth-order moments of $v_{x}$ is presented in Fig. 7. The time trends of the higherorder moments also highlight the presence of the unsteady and quasi-steady phases, with time scales similar to those already identified for the mean kinematic properties of particle motion. Within the unsteady phase, as time increases the particle velocity variance grows while the coefficient of variation, skewness,
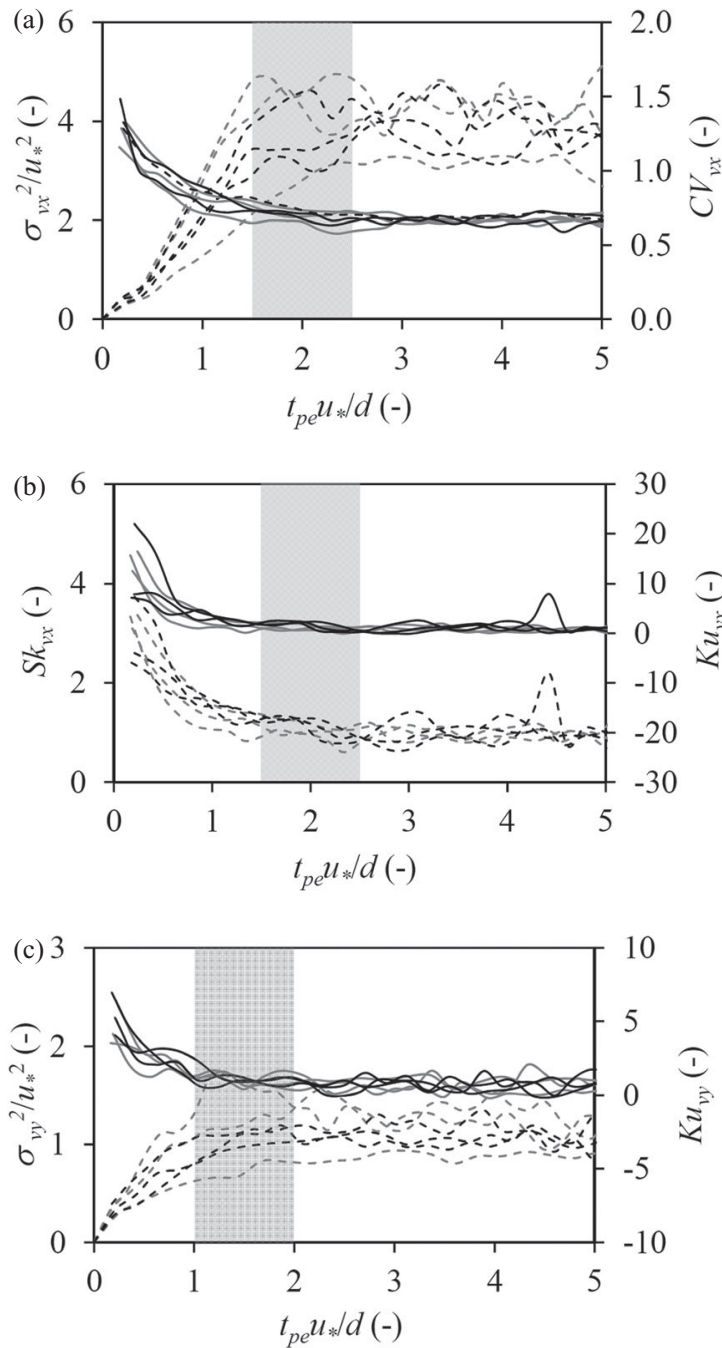

Figure 7 Time evolution of the velocity variance $\sigma_{v i}^{2}$, coefficient of variation $C V_{v i}$, skewness coefficient $S k_{v \mathrm{i}}$, and kurtosis coefficient $K u_{v \mathrm{i}}$ for all tests (FB in grey and MB in black): (a) dimensionless variance (dashed) and coefficient of variation (continuous) for $v_{x}$; (b) skewness (dashed) and kurtosis (continuous) for $v_{x}$; (c) dimensionless variance (dashed) and kurtosis (continuous) for $v_{y}$. The grey strips define a range for dimensionless duration of the unsteady (acceleration) phase $\tau_{a}{ }^{*}$ of particle motion

and kurtosis gradually decrease reflecting a tendency to normality. Figure $7 \mathrm{c}$ shows the evolution of the variance and kurtosis of $v_{y}$ that appears to be similar in shape to that of $v_{x}$ (odd moments for $v_{y}$ are not shown because they are equal to zero at all times). However, the duration of the unsteady phase for the transverse particle velocity is shorter, i.e. $\tau_{a} * \cong 1-2$ compared to $\tau_{a} * \cong 1.5-2.5$ for the streamwise velocity. One may also note in Fig. 7 that the magnitudes of the variance and kurtosis for the transverse velocity within the unsteady phase are appreciably lower than those for the streamwise velocity.

Finally, Figs $6 \mathrm{c}$ and 7 show weak dependence of particle velocity statistics on the bed configuration used in the experiments. 

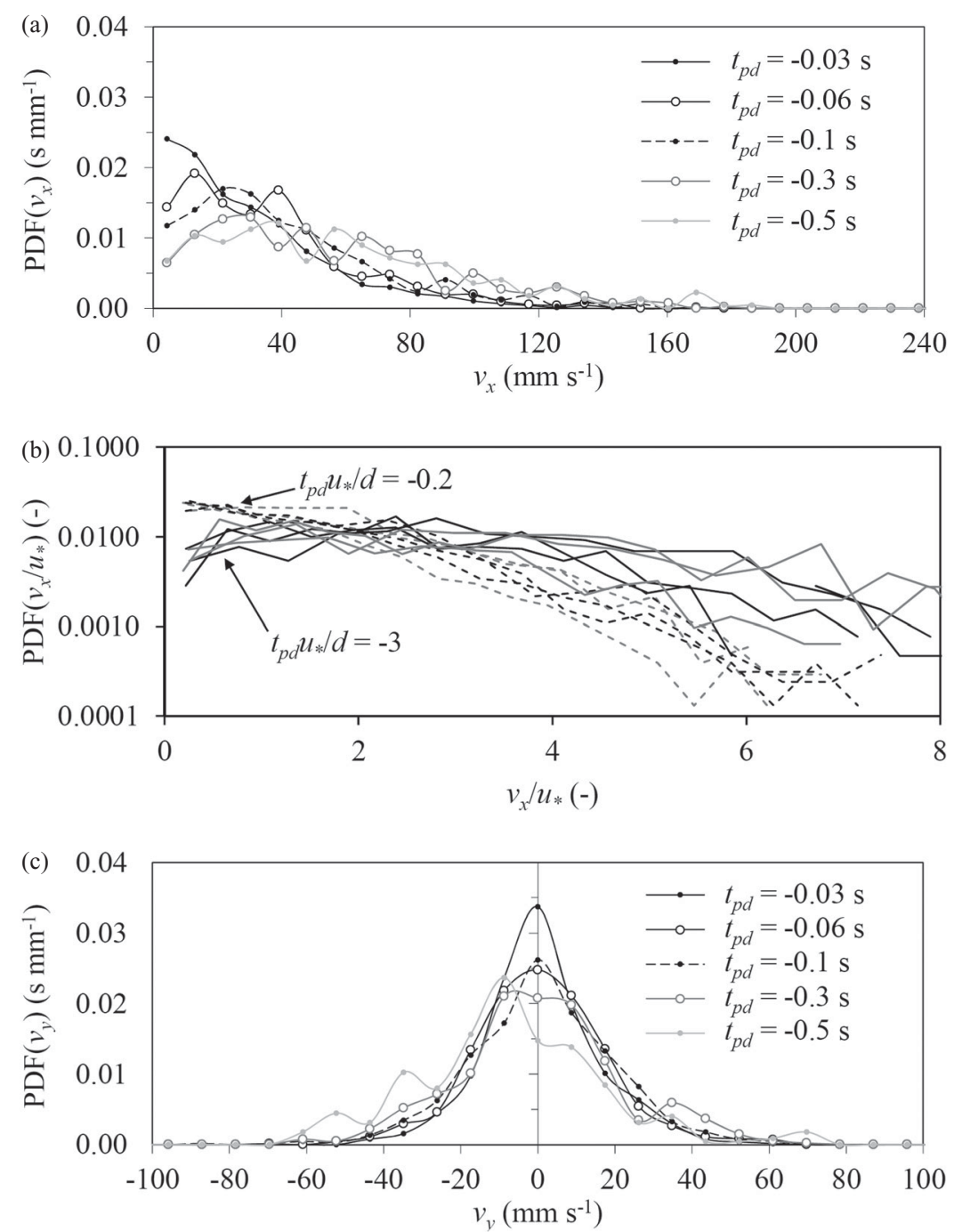

Figure 8 Distributions of instantaneous velocity components at given time instants before disentrainment: (a) probability density function (PDF) of $v_{x}$ for test MB- $Q_{3}$; (b) PDF of $v_{x}$ at $t_{p d} u * / d=-0.2$ (dashed lines) and $t_{p d} u * / d=-3$ (continuous lines) for all tests, FB in grey and MB in black; (c) PDF of $v_{y}$ for test MB- $Q_{3}$

\subsection{Particle kinematics: disentrainment (deceleration) phase}

The mechanics of grain disentrainment in sediment transport studies has received considerably less attention compared to the entrainment mechanics. Below we present particle motion statistics related to this disentrainment phase of particle motion that until now have been missing.

\section{Probability distribution of particle velocity}

The PDFs of instantaneous velocity $v_{x}$ at given time instants before disentrainment are shown in Fig. 8a for test MB-Q $Q_{3}$. As explained in Section 3.1, velocity ensembles in this case are created with samples taken at the same $t_{p d}$. At $t_{p d} \ll 0$ the PDFs correspond to the quasi-steady phase and thus $\operatorname{PDF}\left(t_{p d} \ll 0\right) \cong$ $\operatorname{PDF}\left(t_{p e} \gg 0\right)$. Similar to the situation in the acceleration phase, the probability of low particle velocities decreases with increasing time distance from the disentrainment instant. However, a sharp shift of the distribution mode towards larger velocities occurs at lower time lags than in the acceleration phase. At $t_{p d}=-0.03 \mathrm{~s}$ the distribution is peaked at the origin, with peak probability density being noticeably less than that for entrainment (Fig. 5a), while for lower $t_{p d}$ the mode of the PDF moves to higher velocity values. This observation retains its validity for all tests (Fig. 8b). The PDFs of $v_{y}$ are bell-shaped (Fig. 8c), similar to those for $v_{x}$ (Fig. 5c). As for the PDF of $v_{x}$, for $t_{p d}<-0.03 \mathrm{~s}$ the distributions are similar to each other and the likelihood of vanishing values of particle velocity is lower than at $t_{p d}=-0.03 \mathrm{~s}$.

\section{Statistical moments of particle velocity}

The change of first- to fourth-order moments of $v_{x}$ and even moments of $v_{y}$ before particle disentrainment is depicted in Fig. 9, which gives information similar to that in Fig. 6c and Fig. 7 for the acceleration phase. The velocity history in Fig. 9a shows that, well before disentrainment, a particle moves with a quasi-steady velocity (particle acceleration is nearly zero) as discussed in the previous section (e.g. Fig. 6c). Close to disentrainment, 

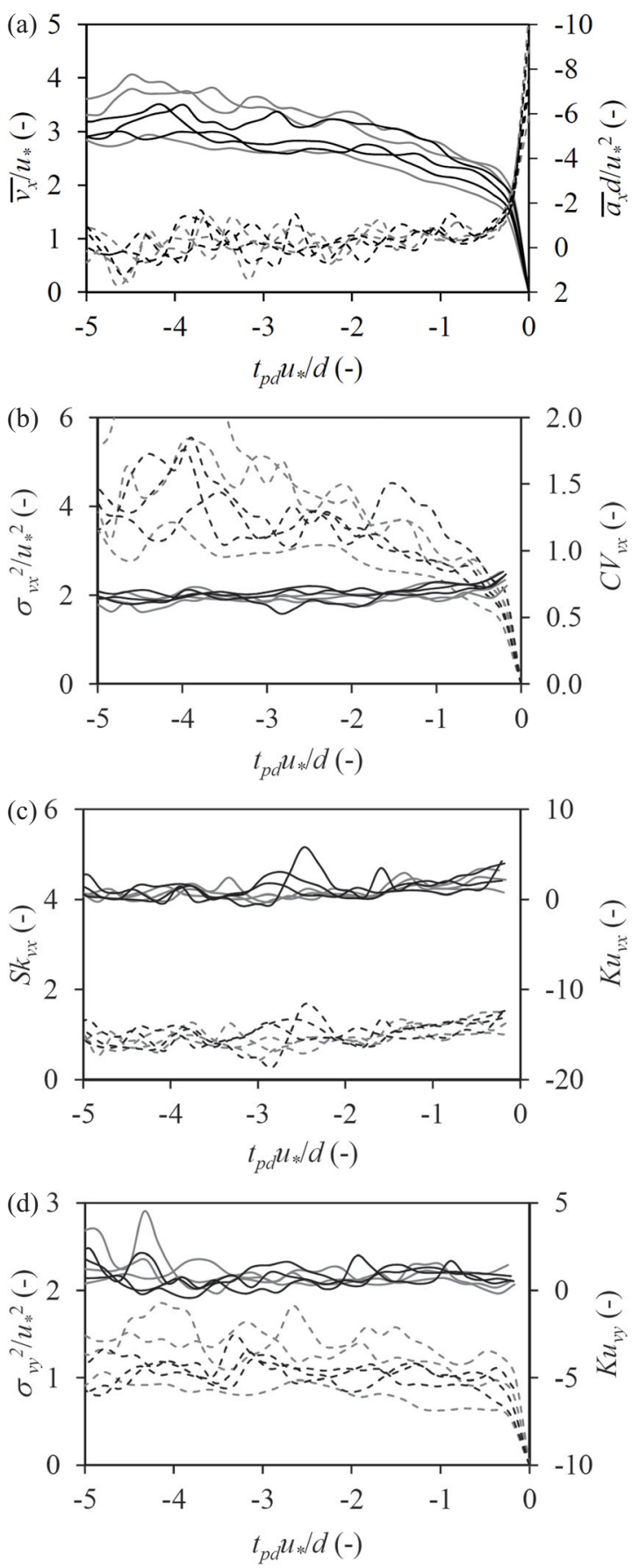

Figure 9 Time evolution of statistical moments of particle velocity components and streamwise acceleration in the disentrainment (deceleration) phase for all tests (FB in grey and MB in black): (a) $\overline{v_{x}} / u_{*}$ (continuous) and $\overline{a_{x}} d / u_{*}^{2}$ (dashed); (b) dimensionless variance $\sigma_{v x}^{2}$ (dashed) and coefficient of variation (continuous) of $v_{x}$; (c) skewness coefficient $S k_{v x}$ (dashed) and kurtosis coefficient $K u_{v x}$ (continuous) of $v_{x}$; and (d) dimensionless variance (dashed) and kurtosis (continuous) of $v_{y}$

starting at $t_{p d} \mathcal{u} * / d \cong-3$ to -4 , gradual decrease in particle velocity is observed (Fig. 9a), followed by a sharp deceleration within the last time step before particle rest, during which the particle loses half of its quasi-steady velocity. For the test $\mathrm{MB}-\mathrm{Q}_{3}$ this final phase corresponds to a displacement of about $0.5 \mathrm{~mm}$, much less than a particle diameter.
The time changes of the second- to fourth-order moments and the coefficient of variation of $v_{x}$, presented in fig. $9 \mathrm{~b}$ and $\mathrm{c}$, are consistent with the description above, qualitatively mir-roring what is found for the acceleration phase after the particle entrainment. The final phase of disentrainment is also visible in the time evolution of the variance of $v_{y}$, shown in Fig. 9d, where an abrupt change in particle behaviour at particle stop is observed. The kurtosis of $v_{y}$, however, does not show any special behaviour at small $\left|t_{p d}\right|$, always being close to 0 .

Overall, similar to the acceleration phase, the disentrain-ment (or deceleration) phase includes two sub-phases: "gradual deceleration" sub-phase and "sharp deceleration" sub-phase. The comparison of Fig. 9 with Figs 6 and 7 suggests that the total dimensionless duration of the deceleration phase, $\tau d^{*}$, is considerably longer than that of the acceleration phase, $\tau_{a} *$.

\subsection{Particle kinematics: conceptual synthesis}

The history of a moving particle can be represented by the time evolution of its velocity from entrainment to disentrainment. Figure 10 summarizes the experimental findings reported in the previous sections and highlights three major phases that can be identified within an intermediate trajectory. The entrained particle first experiences an unsteady acceleration phase (with duration $\tau_{a}$ ), then a quasi-steady phase $\tau_{s}$ long, and finally the disentrainment phase of duration $\tau_{d}$. The acceleration phase can be further split into two sub-phases. During the first sub-phase of duration $\tau_{r}$, the entrained particle moves along a distance comparable to the grain size; its motion in this sub-phase is influenced by the local bed topography. In the second unsteady sub-phase of duration $\left(\tau_{a}-\tau_{r}\right)$ the particle is accelerated by fluid drag up to an equilibrium velocity that is maintained in a following quasi-steady phase of motion. Within this quasisteady phase, the particle interactions with the bed and the turbulent flow are in equilibrium. It should be noted, however, that the ensemble averaging at a particular $\tau_{\text {pe }}$ may involve different phases of trajectories (e.g. quasi-steady and deceleration phases) potentially leading to a pseudo effect of mild deceleration if ensemble-averaged parameters are considered.

After a time $\tau_{a}+\tau_{s}$, the deceleration/disentrainment phase begins. This last phase, which has a total duration of $\tau_{d}$, also includes two sub-phases. In the first one, the grain deceler-ates gradually due to lowering fluid drag effect; we attribute the "gradual-deceleration" sub-phase to a drop in flow veloc-ity due to a passage of a low momentum volume of fluid (e.g. lowmomentum part of a large coherent structure). In the second subphase of duration $\tau_{t}$ the grain finally comes to an abrupt stop in the final "high-deceleration" phase.

\section{Particle diffusion}

\subsection{Experimental results}

The dataset obtained in our experiments enables particle diffusion to be analysed at both the local and intermediate ranges 


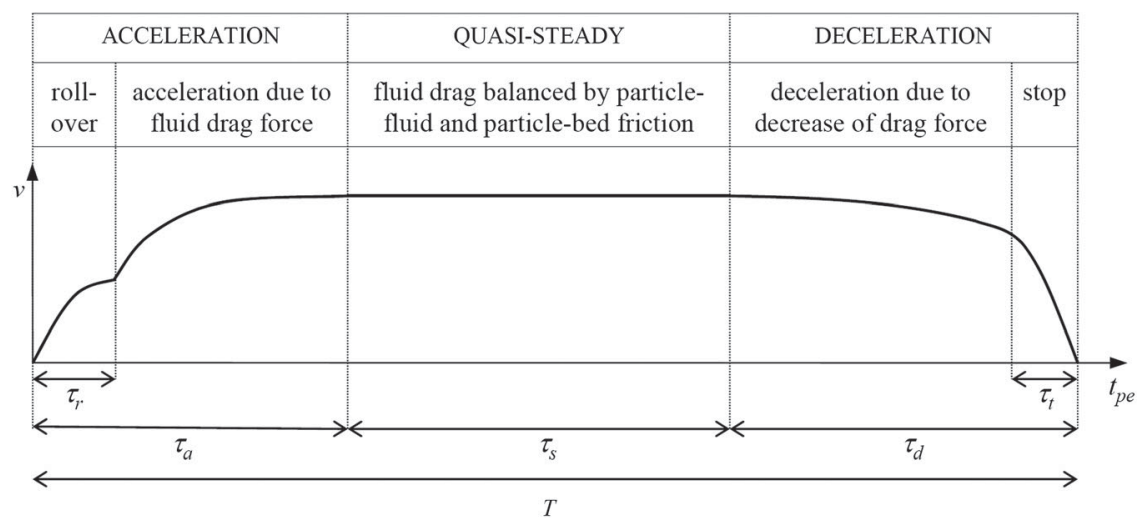

Figure 10 Conceptual representation of particle motion within the intermediate range of scales

of scales (see Introduction for definitions). The global range of scales is not covered here because particle trajectories were measured only from entrainment to disentrainment and are thus limited to the intermediate range.

Figure 11a shows the scaling growth with time of nondimensional variances of streamwise and transverse particle positions, computed for test MB- $Q_{3}$, i.e. $\overline{x_{p e}^{\prime 2}} \propto t_{p e}^{2 \gamma_{x}}$ and $\overline{y_{p e}^{\prime 2}} \propto$ $t_{p e}^{2 \gamma_{y}}$. The starting point was set at the particle entrainment as indicated by subscripts (see Section 3.1 and Fig. 4). In our analysis we studied local values of exponents $\gamma_{x}$ and $\gamma_{y}$, computed as local slopes of the scaling curves at particular travel times. The local values of $\gamma_{x}$ and $\gamma_{y}$ provide more details on the time evolution of the variances compared to the traditional approach where the scaling exponents are obtained as "least-square averages" over the whole scaling range. In the acceleration phase when $t_{p e} u * / d<\tau_{a} *$, the local growth rate for the $x$-direction (i.e. $\gamma_{x}$ ) is higher than that within the quasi-steady phase at $t_{p e} u * / d>\tau_{a} *$. Initially, the scaling exponent $\gamma_{x}$ increases from 1.0 to 1.5 and then reduces again (Fig. 11a). Along the transition from the acceleration phase to the quasi-steady phase the value of $\gamma_{x}$ gradually decreases and, for the largest observable diffusion times, it is in general within the range $0.5<\gamma_{x}<1$ (still indicating super-diffusion). In the transverse direction, the local exponent $\gamma_{y}$ is around 1.0 at small diffusion times within the acceleration phase, reducing to $\gamma_{y}=0.5-1.0$ at larger times of the steady-state phase. The results reported above for MB- $Q_{3}$ are similar for all other tests, as shown in Fig. 11b (limited to the streamwise component for the sake of clarity).

As discussed in Ballio, Campagnol, Nikora, and Radice (2013), values for $\gamma$ larger than 1.0, unexpected within the conceptual framework of Nikora et al. (2001, 2002), can be explained by the acceleration experienced by the particles during the initial unsteady phase of motion. While for a cloud of grains moving with random constant velocities the variance of particle coordinates would grow as $\overline{x_{p}^{\prime 2}} \propto t_{p}^{2}$ (corresponding to $\gamma_{x}=1$, ballistic behaviour), a cloud of grains with random constant acceleration would diffuse as $\overline{x_{p}^{\prime 2}} \propto t_{p}^{4}\left(\gamma_{x}=2\right.$, "superballistic" behaviour). Full derivation is given in Ballio et al. (2013) and briefly reported in Appendix 1. In reality, as was
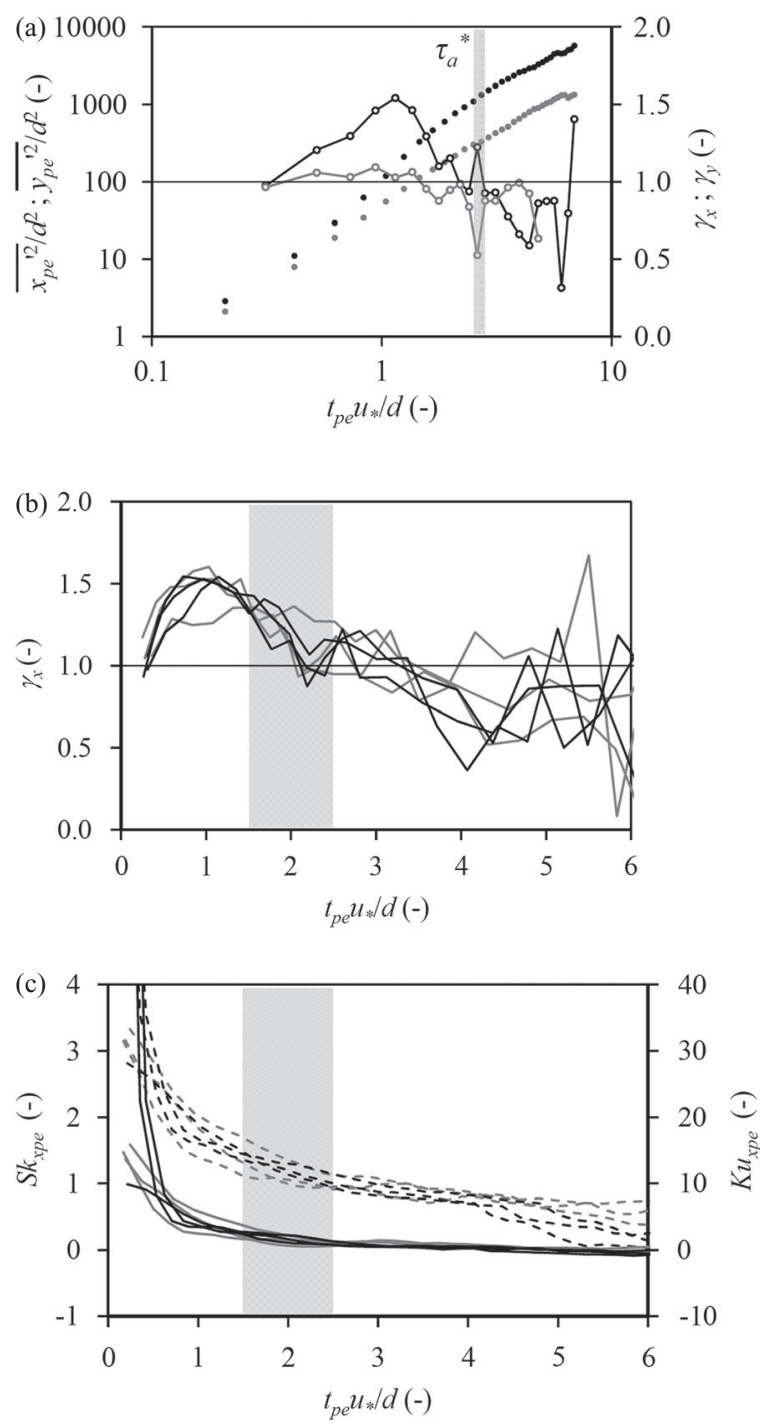

Figure 11 Particle diffusion statistics: (a) growth in time of variances of particle position (filled symbols) and related local scaling exponents (hollow symbols) for MB- $Q_{3}$ (streamwise and transverse directions in black and grey, respectively); (b) local scaling exponents characterizing diffusion along the streamwise direction for all tests (FB in grey and MB in black); (c) time evolution of skewness coefficient $S k_{x p e}$ (dashed) and kurtosis coefficient $K u_{x p e}$ (continuous) for all tests (FB in grey and $\mathrm{MB}$ in black). The grey strips (as in Figs 6 and 7) define a range of dimensionless duration of the acceleration phase $\tau_{a}{ }^{*}$ of particle motion 


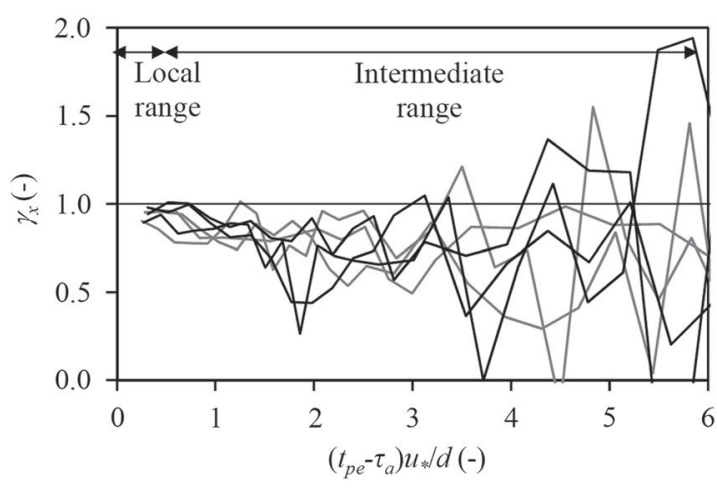

Figure 12 Local scaling exponents characterizing diffusion along the streamwise direction for all tests (FB in grey and MB in black). Only the quasi-steady phase of motion was considered in the scaling analysis, i.e. acceleration and deceleration phases have been removed from the data

shown in the previous sections, particle acceleration decreases with time and this explains experimental values $1<\gamma_{x}<2$ during the acceleration phase.

The higher-order moments of the streamwise particle position (skewness and kurtosis coefficients) are shown in Fig. 11c for all tests. The obtained values of $S k_{x p e}\left(t_{p e}\right)$ and $K u_{x p e}\left(t_{p e}\right)$ deviate from Gaussian values (which should be zero) at small times, tending to Gaussian behaviour at large diffusion times. Once again, the unsteadiness of motion in the acceleration phase strongly influences the dispersion process.

To compare our measurements with the standard dispersion theory, which does not consider unsteadiness effects, we repeated the scaling analysis removing the acceleration phase $\left(t_{p e}>\tau_{a}\right)$ and final "high-deceleration" sub-phase $\left(\left|t_{p d}\right|>\tau_{t}\right)$ from the data. A more rigorous selection of the quasi-steady phase would have required to cut off the whole deceleration phase $\left(\left|t_{p d}\right|>\tau_{d}\right.$, Fig. 10) but we resolved to preserve the "gradual-deceleration" sub-phase for the sake of a larger sample of reduced trajectories. Results of this exercise are given in Fig. 12. At short diffusion times, the diffusive behaviour is now ballistic $\left(\gamma_{x}=1\right)$, with the local range extending up to $\left(t_{p e}-\tau\right.$ a) $u * / d=0.5$. The corresponding length limiting the local range is of the order of $d$, which is comparable to the prevailing scale of the rolling mechanism. At longer diffusion times, particle diffusion retrieves the super-diffusive behaviour as in Fig. $11 \mathrm{~b}\left(0.5<\gamma_{x}<1\right)$. Similar results were obtained by Nikora et al. (2002) and R.L. Martin et al. (2012) for diffusion of sorted gravel moving as bed load. Such a super-diffusive behaviour may be related to turbulence effects enhancing par-ticle dispersion, as shown by numerical simulations of Bialik et al. (2012).

Comparison of Figs $11 \mathrm{~b}$ and 12 underlines the strong influence of the unsteady phase of particle motion on its diffusion properties. The "super-ballistic" scaling behaviour at short times was first noted, although not explained, by Bialik et al. (2012) who modelled particle saltation in a turbulent flow.
The authors found that when the starting points of trajecto-ries were defined at sediment entrainment, particle diffusion at short times ( $t_{p e} u_{*} / d<3$ ) could be strongly enhanced by the particular entrainment mechanism identified as a "near-field effect".

\subsection{Refined diffusion model for particle motion}

Nikora et al.'s $(2001,2002)$ conceptual model was developed to describe a diffusive process resulting from homogeneous motion of particles. Such motion is, in general, characterized by random accelerations/decelerations along trajectories as a result of the fluid-particle, particle-bed, and particle-particle interactions (the last process was negligible for the experimental conditions of the present work). The overall diffusive process is a conse-quence of the combination of such random processes. In this paper it has been shown, however, that the local and interme-diate trajectories are not homogeneous and include two phases of unsteady motion which cannot be neglected and should be accounted for in the diffusion model. In this section we discuss how these findings can be included in a conceptual model for particle diffusion.

Let us start considering the effects of the acceleration phase. In general, at least two different approaches can be used for the analysis of particle diffusion. Within the first (conventional) approach, an ensemble of moving particle trajectories is formed by superimposing randomly selected coordinates and considering the latter as starting points corresponding to $t_{p}=0, x_{p}=0$, and $y_{p}=0$. The starting coordinates may include, but it is not the most frequent case, instances of entrainment. This approach was essentially followed in Nikora et al. (2002). According to the second approach, an ensemble of particle trajectories is formed by superimposing particle coordinates corresponding to the entrainment instances only. Both approaches are equally applicable for sediment transport studies highlighting different facets of sediment dynamics. For example, the first approach may be more relevant for description of diffusion effects on sediment fluxes, while the second approach may be more suitable for describing tracer particles.

If the first approach is followed, the initial points will be randomly distributed along the different phases indicated in Fig. 10 (acceleration after entrainment, quasi-steady, decel-eration before disentrainment). For fairly long intermediate trajectories with the steady-state phase dominant, no specific behaviour linked to the unsteady phases would emerge. In other words, the diffusion pattern should appear as that in Fig. 12, where the initial phase was artificially removed, and a ballistic behaviour $(\gamma=1)$ became clear at small time scales. However, in the case of short intermediate trajectories (weak transport conditions), where the acceleration phase covers a large fraction of the trajectory, diffusion coefficients larger than 1 may appear at the local range due to the high probability of starting points being within the acceleration phase. If the second approach is followed then the local range will start with "super-ballistic" 


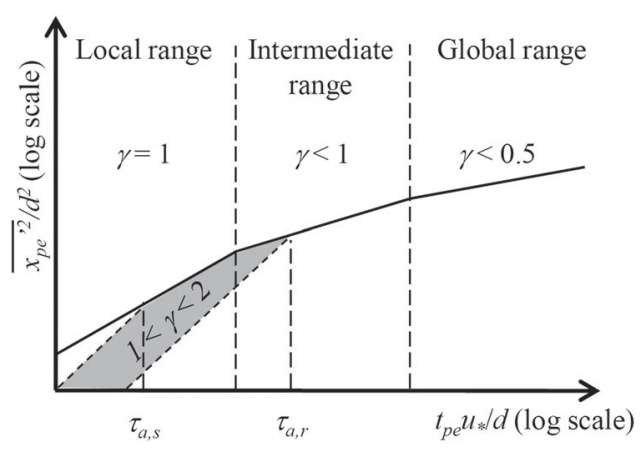

Figure 13 Refined conceptual model of sediment dispersion accounting for entrainment-related unsteadiness of particle motion. The particle acceleration (grey range) may completely mask the (ballistic) local range. $\tau_{a, r}$ is the duration of acceleration phase for a rolling (or sliding) particle; $\tau_{a, s}$ is the duration of acceleration phase for a saltating particle

$(\gamma>1)$ subrange, as a result of particle acceleration within the initial unsteady phase. Consequently, an additional line should be added in the diffusion model of Fig. 1, as shown in Fig. 13. A dashed line accounting for particle unsteadiness may be moved left- or right-wards depending on the time scale of the acceler-ation $\tau_{a}$ compared to the conventional time scale for the local range. If the acceleration phase covers a major part of the trajec-tory, any ballistic behaviour would be more or less masked by the "super-ballistic" behaviour due to acceleration (as it was in our tests, Fig. 11).

The extension of $\tau_{a}$ within the motion ranges shall depend on the transport conditions. In particular, for prevailing rolling motion we may expect the time scale for the local range to be comparable to or smaller than that of the acceleration phase ( $\tau_{a, r}$ in Fig. 13), while for prevailing saltating motion the acceleration phase would affect only a (small) part of the local trajectory, so that the full sequence of behaviours should be identifiable $\left(\tau_{a, s}\right.$ in Fig. 13). The global range should not be influenced by "super-ballistic" behaviour as $\tau_{a}$ is always shorter than the duration of intermediate trajectories.

The effect of the disentrainment phase on the particle dispersion is expected to be much less profound compared to the effect of the acceleration phase. Indeed, due to a wide distribution of trajectory durations (Fig. 3) the disentrainment phases are always "mixed" with steady-state parts of particle trajectories when ensemble averaging is performed, irrespective of approaches used. In addition, the typical fraction of the "highdeceleration" sub-phase within the intermediate trajectory is fairly small (much less than $d$ ) while the unsteadiness within the "gradual-deceleration" sub-phase is weak.

\section{Conclusions}

This paper presented a novel dataset and its conceptual interpretation in relation to bed particle motion within its intermediate trajectory, defined as particle motion between instants of entrainment and disentrainment. The experiments involved high-resolution measurements of particle motion for weak bed-load conditions and a range of flow rates up to 1.3 times the threshold rate. The data analysis focused on the instantaneous data of particle motion to explore: (1) ensembleaveraged time evolution of particle velocity and acceleration; (2) high-order statistical moments and PDF of grain velocity; and (3) time evolution of dispersion characteristics including variance, skewness, and kurtosis of particle positions. The key results of this work can be summarized as follows.

- The intermediate particle trajectory can be presented as a sequence of three major phases of particle motion: (1) initial unsteady phase after entrainment that can be defined as the acceleration phase; (2) quasi-steady phase; and (3) final unsteady phase, prior to disentrainment, that can be defined as the deceleration phase. The high-resolution measurements enabled the acceleration phase to be split into two sub-phases, a "rollover" sub-phase and a "reducing acceleration" subphase. In a similar way, the final deceleration phase could be split into a "gradual-deceleration" sub-phase and a "highdeceleration" sub-phase. The physical mechanisms behind the sub-phases are different and may depend on the specific details of particle-flow, particle-bed, and particle-particle interactions. A physically-based conceptual model of particle trajectory was proposed accordingly.

- It was shown that particle unsteadiness can give rise to diffusion exponents larger than that for ballistic motion, assumed for a local range of scales. A refined conceptual model of particle diffusion was proposed accounting for the effect of the acceleration phase. The new conceptual picture combines the diffusion model proposed by Nikora et al. (2001, 2002), assuming homogeneity of particle motion, and the threephase structure of intermediate particle trajectory, introduced in this paper.

- The key statistical properties of particle trajectories appear to be weakly affected by the transport capacity of the flow, at least within the range of investigated experimental conditions.

- It is proposed that the succession of diffusion scales might depend on the motion mode of particles (rolling, sliding, or saltation). However, the experimental methodology used in our study did not enable the particle motion mode to be unambiguously recognized and thus confirmation of this assumption will be left to follow-up studies.

\section{Acknowledgements}

The authors wish to thank Roger Nokes for constant support on image processing by Streams software, and Aleksandra Lescova and Viktoriya Bulankina for performing the experiments within their MSc theses. 


\section{Funding}

This work was supported by the Research Executive Agency, through the 7th Framework Programme of the European Union, Support for Training and Career Development of Researchers [Marie Curie - FP7-PEOPLE-2012-ITN], which funded the Initial Training Network (ITN) HYTECH "Hydrodynamic Transport in Ecologically Critical Heterogeneous Interfaces" [number 316546]. It was also partially supported by the Italian Ministry of University and Research and by the UK EPSRC [grant number EP/G056404/1].

\section{Supplemental data}

The supplemental data for this paper is available online at http://dx.doi.org/10.1080/00221686.2015.1085920.

A detailed description of the previous theoretical, numerical and experimental findings on sediment diffusion is synthesized in two tables.

The first table collects the main characteristics of the studies: research methodology (numerical, field or laboratory tests), considered range of scales (local, intermediate or global), bed and grain properties, Shields number, and submergence. In the second table, for each work and each range of scales the following are shown: observed kind of diffusion; mathematical evidence of normal or anomalous diffusion; and physical reason for normal or anomalous diffusion.

\section{Appendix 1 Derivation of variance scaling growth of particle coordinates}

Let us consider a cloud of grains released in a flow at the same time in the same point. Supposing that each particle moves with a constant streamwise velocity (ballistic behaviour), the total streamwise distance covered by the $\mathrm{n}^{\text {th }}$ particle with a streamwise velocity $v_{x \mathrm{n}}$, increases as $x_{p \mathrm{n}}\left(t_{p}\right)=v_{x \mathrm{n}} t_{p}$. Therefore, the mean position of the cloud centre at time $t_{p}$ is:

$$
\overline{x_{p}}\left(t_{p}\right)=\frac{1}{N} \sum_{\mathrm{n}=1}^{N} v_{x \mathrm{n}} t_{p}=\left(\frac{1}{N} \sum_{\mathrm{n}=1}^{N} v_{x \mathrm{n}}\right) t_{p}=\overline{v_{x}} t_{p}
$$

while the variance of streamwise particles coordinates at time $t_{p}$ is:

$$
\begin{aligned}
\overline{x_{p}^{\prime 2}} & =\frac{1}{N} \sum_{\mathrm{n}=1}^{N}\left[x_{p \mathrm{n}}\left(t_{p}\right)-\overline{x_{p}}\left(t_{p}\right)\right]^{2}=\frac{1}{N} \sum_{\mathrm{n}=1}^{N}\left(v_{x \mathrm{n}} t_{p}-\overline{v_{x}} t_{p}\right)^{2} \\
& =\left(\frac{1}{N} \sum_{\mathrm{n}=1}^{N} v_{x \mathrm{n}}^{2}-2 \overline{v_{x}} \sum_{\mathrm{n}=1}^{N} v_{x \mathrm{n}}+{\overline{v_{x}}}^{2}\right) t_{p}^{2}=\left(\overline{v_{x}^{2}}-{\overline{v_{x}}}^{2}\right) t_{p}^{2}
\end{aligned}
$$

For this scenario the variance of the streamwise particle coordinate grows as $\overline{x_{p}^{\prime 2}} \propto t_{p}^{2}$, resulting in a ballistic diffusion $\left(\gamma_{\mathrm{x}}=1\right)$.

If the particles are uniformly accelerated (each with different acceleration) and $a_{x \mathrm{n}}$ is the streamwise acceleration of the $\mathrm{n}^{\text {th }}$ particle, the total distance covered by the $\mathrm{n}^{\text {th }}$ grain along the streamwise direction increases as $x_{p \mathrm{n}}\left(t_{p}\right)=0.5 a_{x \mathrm{n}} t_{p}{ }^{2}$. The mean position of the cloud centre at time $t_{p}$ is:

$$
\overline{x_{p}}\left(t_{p}\right)=\frac{0.5}{N} \sum_{\mathrm{n}=1}^{N} a_{x \mathrm{n}} t_{p}^{2}=0.5\left(\frac{1}{N} \sum_{\mathrm{n}=1}^{N} a_{x \mathrm{n}}\right) t_{p}^{2}=0.5 \overline{a_{x}} t_{p}^{2}
$$

while the variance of streamwise particles coordinates at time $t_{p}$ can be computed as:

$$
\begin{aligned}
\overline{x_{p}^{\prime 2}} & =\frac{1}{N} \sum_{\mathrm{n}=1}^{N}\left[x_{p \mathrm{n}}\left(t_{p}\right)-\overline{x_{p}}\left(t_{p}\right)\right]^{2} \\
& =\frac{1}{N} \sum_{\mathrm{n}=1}^{N}\left(0.5 a_{x \mathrm{n}} t_{p}^{2}-0.5 \overline{a_{x}} t_{p}^{2}\right)^{2} \\
& =\left(0.25 \frac{1}{N} \sum_{\mathrm{n}=1}^{N} a_{x \mathrm{n}}^{2}-0.5 \overline{a_{x}} \sum_{\mathrm{n}=1}^{N} a_{x \mathrm{n}}+0.25{\overline{a_{x}}}^{2}\right) t_{p}^{4} \\
& =0.25\left(\overline{a_{x}^{2}}-{\overline{a_{x}}}^{2}\right) t_{p}^{4}
\end{aligned}
$$

Thus, if all the grains are accelerated in the streamwise direction the variance of particle coordinates will grow as $\overline{x_{p}^{\prime 2}} \propto t_{p}^{4}$ $\left(\gamma_{\mathrm{x}}=2\right)$

\section{Notation}

$\mathrm{FB}, \mathrm{MB}=$ fixed and mobile bed conditions

$\bar{Z} \quad=$ ensemble average of a generic variable $Z$

$\sigma_{Z} \quad=$ standard deviation of a generic variable $Z$

$C V_{\mathrm{Z}}=\sigma_{Z} / \bar{Z}=$ coefficient of variation of a generic variable $Z$

$S k_{Z} \quad=$ skewness coefficient of a generic variable $Z$, $S k_{Z}=\overline{(Z-\bar{Z})^{3}} / \sigma_{Z}^{3}$

$K u_{Z} \quad=$ kurtosis coefficient of a generic variable $Z$, $\left.K u_{Z}=\overline{(Z-\bar{Z})}\right)^{4} / \sigma_{Z}^{4}-3$

$a_{\mathrm{x}} \quad=$ streamwise component of instantaneous particle acceleration $\left(\mathrm{cm} \mathrm{s}^{-2}\right)$

$d \quad=$ equivalent diameter of moving grains $(\mathrm{mm})$

$g \quad=$ gravity acceleration $\left(\mathrm{m} \mathrm{s}^{-2}\right)$

$\mathscr{L}=$ distance travelled by one grain along its intermediate trajectory $(\mathrm{mm})$

$\mathscr{L}_{p e} \quad=$ distance travelled by one grain from entrainment (mm)

$L_{x} \quad=$ streamwise component of $\mathscr{L}(\mathrm{mm})$

$L_{\text {xpe }} \quad=$ streamwise component of $\mathscr{L}_{\text {pe }}(\mathrm{mm})$ 


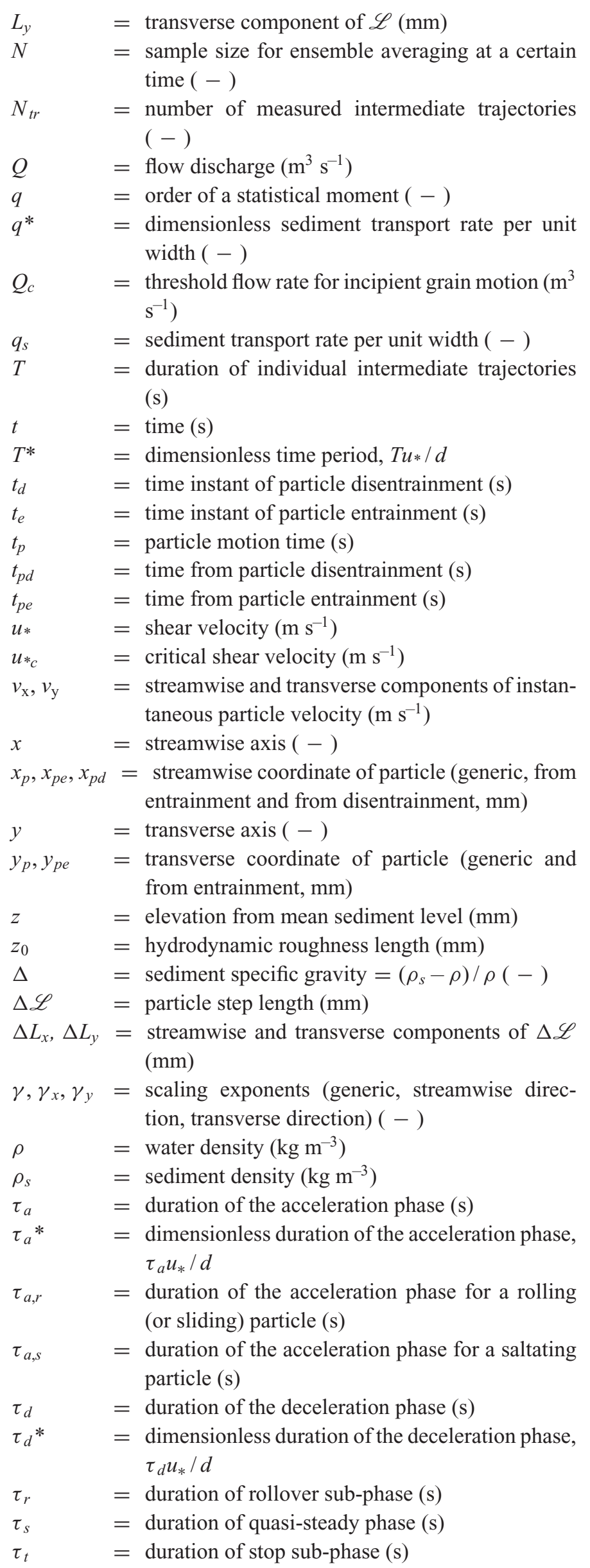

\section{References}

Ancey, C., Davison, A. C., Böhm, T., Jodeau, M., \& Frey, P. (2008). Entrainment and motion of coarse particles in a shallow water stream down a steep slope. Journal of Fluid Mechanics, 595, 83-114.

Ballio, F., Campagnol, J., Nikora, V., \& Radice, A. (2013). Diffusive properties of bed load moving sediments at short time scales. Proceedings of the XXXV IAHR Congress, Chengdu, China.

Ballio, F., \& Tait, S. (2012). Sediment transport mechanics. Acta Geophysica, 60, 1493-1499.

Bialik, R. J. (2011). Numerical study of saltation of non-uniform grains. Journal of Hydraulic Research, 49, 697-701.

Bialik, R. J., Nikora, V., \& Rowiński, P. M. (2012). 3D Lagrangian modelling of saltating particles diffusion in turbulent water flow. Acta Geophysica, 60, 1639-1660.

Bouchaud, J. P., \& Georges, A. (1990). Anomalous diffusion in disordered media: Statistical mechanisms, models and physical applications. Physics Reports - Review Section of Physics Letters, 195, 127-293.

Bradley, D. N., Tucker, G. E., \& Benson, D. A. (2010). Fractional dispersion in sand bed river. Journal of Geophysical Research - Earth Surface, 115, F00A09, doi:10.1029/ 2009JF001268.

Campagnol, J., Radice, A., \& Ballio, F. (2012a). Insight on how bed configuration affects properties of bed load motion. Proceedings of ICSE 2012, Sixth International Conference on Scour and Erosion, Paris, France.

Campagnol, J., Radice, A., \& Ballio, F. (2012b). Scale-based statistical analysis of sediment fluxes. Acta Geophysica, 60, 1744-1777.

Campagnol, J., Radice, A., Nokes, R., Bulankina, V., Lescova, A., \& Ballio, F. (2013). Lagrangian analysis of bed-load sediment motion: database contribution. Journal of Hydraulic Research, 51, 589-596.

Drake, T. G., Shreve, R. L., Dietrich, W. E., Whiting, P. J., \& Leopold, L. B. (1988). Bedload transport of fine gravel observed by motion-picture photography. Journal of Fluid Mechanics, 192, 193-217.

Fan, N., Zhong, D., Wu, B., Foufoula-Georgiou, E., \& Guala, M., (2014). A mechanistic-stochastic formulation of bed load particle motions: From individual particle forces to the Fokker-Planck equation under low transport rates. Journal of Geophysical Research - Earth Surface, 119, 464 482.

Foufoula-Georgiou, E., \& Stark, C. (2010). Introduction to special section on stochastic transport and emergent scaling on Earth's surface: rethinking geomorphic transport - stochastic theories, broad scales of motion and nonlocality. Journal of Geophysical Research - Earth Surface, 115, F00A01, doi:10.1029/2010JF001661.

Francis, J. R. D. (1973). Experiments on the motion of solitary grains along the bed of a water-stream. Proceedings of the Royal Society of London, A, 332, 443-471. 
Furbish, D. J., Ball, A. E., \& Schmeeckle, M. W. (2012). A probabilistic description of the bed load sediment flux: 4 . Fickian diffusion at low transport rates, Journal of Geophysical Research - Earth Surface, 117, F03034, doi:10.1029/ 2012JF002356.

Furbish, D. J., Haff, P. K., Roseberry, J. C., \& Schmeeckle, M. W. (2012). A probabilistic description of the bed load sediment flux: 1. Theory. Journal of Geophysical Research Earth Surface, 117, F03031, doi:10.1029/2012JF002352.

Furbish, D. J., Roseberry, J. C., \& Schmeeckle, M. W. (2012). A probabilistic description of the bed load sediment flux: 3 . The particle velocity distribution and the diffusive flux. Journal of Geophysical Research - Earth Surface, 117, F03033, doi:10.1029/2012JF002355.

Furbish, D. J., \& Schmeeckle, M. W. (2013). A probabilistic derivation of the exponential-like distribution of bed load par-ticle velocities. Water Resources Research, 49,1537-

Goln56, B., \& Church, M. (1989). An assessment of bed load sediment transport formulae for gravel bed rivers. Water Resources Research, 25, 1161-1186.

Hassan, M., Voepel, H., Schumer, R., Parker, G., \& Fraccarollo, L. (2013). Displacement characteristics of coarse fluvial bed sediments. Journal of Geophysical Research - Earth Surface, 118, 155-165.

Havlin, S., \& Ben-Avraham, D. (2002). Diffusion in disordered media. Advances in Physics, 51, 187-292.

Heyman, J., \& Ancey, C. (2014). Tracking bedload

particdesteep flume: methods and results. Proceedings of River Flow 2014, Seventh International Conference on Fluvial Hydraulics, Lausanne, Switzerland.

Hu, C., \& Hui, Y. (1996). Bed-load transport. I: Mechanical characteristics. Journal of Hydraulic Engineering, 122, 245-254.

Julien, P. Y., \& Bounvilay, B. (2013). Velocity of rolling bed load particles. Journal of Hydraulic Engineering, 139, 177-186.

Lajeunesse, E., Malverti, L., \& Charru, F. (2010). Bedload transport in turbulent flow at the grain scale: experiments and modeling. Journal of Geophysical Research - Earth Surface, 115, F04001, doi:10.1029/2009JF001628.

Lee, H. Y., \& Hsu, I. S. (1994). Investigation of saltating particle motions. Journal of Hydraulic Engineering, 120, 831-845, doi:10.1061/(ASCE)0733-9429(1994)120:7(831).

Martin, R. L., Jerolmack, D. J., \& Shumer, R. (2012). The physical basis of anomalous diffusion in bed load trans-port. Journal of Geophysical Research - Earth Surface, 117, F01018, doi:10.1029/2011JF002075.

Martin, Y. (2003). Evaluation of bed load transport formulae using field evidence from the Vedder River, British

Columbia. Geomorphology, 53, 75-95.

McCuen, R. H. (1993). Microcomputer applications in statistical hydrology. Englewood Cliffs, NJ: Prentice Hall.

Metzler, R., \& Klafter, J. (2004). The restaurant at the end of the random walk: recent developments in the description of anomalous transport by fractional dynamics. Journal of Physics A - Mathematical and General, 37, R161-R208, doi:10.1088/0305-4470/37/31/R01.

Nikora, V. I., Goring, D. G., \& Biggs, B. J. F. (1998). On gravelbed roughness characterization. Water Resources Research, 34, 517-527.

Nikora, V., Habersack, H., Huber, T., \& McEwan, I. (2002). On bed particle diffusion in gravel bed flows under weak bed load transport. Water Resources Research, 38, 171-179.

Nikora, V., Heald, J., Goring, D., \& McEwan, I. (2001). Diffusion of saltating particles in unidirectional water flow over a rough granular bed. Journal of Physics A-Mathemati-cal and General, 34, L743-L749, doi:10.1088/0305-4470/34/ $50 / 103$.

Niño, Y., \& Garcìa, M. (1998). Experiments on saltation of sand in water. Journal of Hydraulic Engineering, 124, 1014-1025.

Niño, Y., Garcìa, M., \& Ayala, L. (1994). Gravel saltation 1. experiments. Water Resources Research, 30, 1907-1914.

Radice, A., \& Ballio, F. (2008). Double-average characteristics of sediment motion in one-dimensional bed load. Acta Geophysica, 56, 654-668, doi:10.2478/s11600-008-0015-0.

Radice, A., Ballio, F., \& Nikora, V. (2010). Statistics and characteristic scales for bed load in a channel flow with sidewall effects. Acta Geophysica, 58, 1072-1093.

Ramesh, B., Kothyari, U. C., \& Murugesan, K. (2011). Nearbed particle motion over transitionally-rough bed. Journal of Hydraulic Research, 49, 757-765.

Recking, A. (2013). An analysis of nonlinearity effects on bed load transport prediction. Journal of Geophysical Research -Earth Surface, 118, 1264-1281.

Roseberry, J. C., Schmeeckle, M. W., \& Furbish, D. J. (2012). A probabilistic description of the bed load sediment flux: 2. Par-ticle activity and motions. Journal of Geophysical Research

- Earth Surface, 117, F03032, doi:10.1029/2012JF002353.

Samson, L., Ippolito, I., Bideau, D., \& Batrouni, G. G. (1999). Motion of grains down a bumpy surface. Chaos, 9, 639-648. Sayre, W., \& Hubbell, D. (1965). Transport and dispersion of labelled bed material, North Loup River, Nebraska. U.S. Geological Survey Professional Papers, 433-C.

Viswanathan, G. M., Raposo, E. P., Bartumeus, F., Catalan, J., \& da Luz, M. G. E. (2005). Necessary criterion for distinguishing true superdiffusion from correlated random walk processes. Physical Review E, 72, 011111, doi:10.1103/ PhysRevE.72.011111.

Wang, K. G. (1994). Long-range correlation effects, generalised Brownian motion and anomalous diffusion. Journal of Physics A-Mathematical and General, 27, 3655-3661.

Weeks, E. R., Urbach, J. S., \& Swinney, H. L. (1996). Anomalous diffusion in asymmetric random walks with a quasigeostrophic flow example. Physica D-Nonlinear Phenomena, 97, 291-310.

Zhang, Y., Meerschaert, M. M., \& Packman, A. I. (2012). Linking fluvial bed sediment transport across scales. Geophysical Research Letters, 39, L20404, doi:10.1029/2012GL053476. 